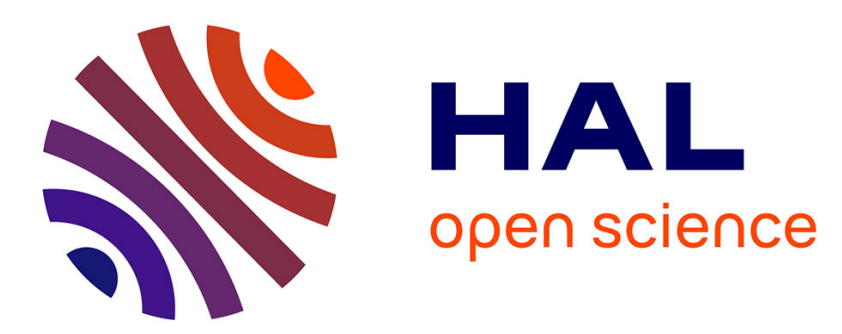

\title{
Smart Responsive Polymers: Fundamentals and Design Principles
}

Debashish Mukherji, Carlos Marques, Kurt Kremer

\section{To cite this version:}

Debashish Mukherji, Carlos Marques, Kurt Kremer. Smart Responsive Polymers: Fundamentals and Design Principles. Annual Review of Condensed Matter Physics, 2020, 11 (1), pp.271-299. 10.1146/annurev-conmatphys-031119-050618 . hal-03100989

\section{HAL Id: hal-03100989 \\ https://hal.science/hal-03100989}

Submitted on 6 Jan 2021

HAL is a multi-disciplinary open access archive for the deposit and dissemination of scientific research documents, whether they are published or not. The documents may come from teaching and research institutions in France or abroad, or from public or private research centers.
L'archive ouverte pluridisciplinaire HAL, est destinée au dépôt et à la diffusion de documents scientifiques de niveau recherche, publiés ou non, émanant des établissements d'enseignement et de recherche français ou étrangers, des laboratoires publics ou privés. 


\title{
Smart responsive polymers: Fundamentals and design principles
}

\section{Debashish Mukherji, ${ }^{1,2}$ Carlos M. Marques, ${ }^{3}$ and Kurt Kremer ${ }^{2}$}

\author{
${ }^{1}$ Stewart Blusson Quantum Matter Institute, University of British Columbia, \\ Vancouver BC V6T 1Z4, Canada: \\ debashish.mukherji@ubc.ca;mukherji@mpip-mainz.mpg.de \\ ${ }^{2}$ Max-Planck Institut für Polymerforschung, Ackermannweg 10, 55128 Mainz \\ Germany: kremer@mpip-mainz.mpg.de \\ ${ }^{3}$ Institut Charles Sadron, Université de Strasbourg, CNRS, Strasbourg, France: \\ marques@unistra.fr
}

Xxxx. Xxx. Xxx. Xxx. YYYY. AA:1-29

https://doi.org/10.1146/((please add article doi))

Copyright (c) YYYY by Annual Reviews. All rights reserved

\section{Keywords}

Soft matter, Smart polymers, Multi-responsive systems, Solvation thermodynamics

\section{Abstract}

In this review we summarize recent theoretical and computational developments in the field of smart responsive materials, together with complementary experimental data. A material is referred to as smart responsive when a slight change in external stimulus can drastically alter its structure, function, or stability. Because of this smart responsiveness, these systems are used for the design of advanced functional materials. The most characteristic properties of smart polymers will be discussed, especially polymer properties in solvent mixtures. We will show how a multi-scale simulation approach can shed light on the intriguing experimental observations. Special emphasis will be given to two symmetric phenomena: co-non-solvency and co-solvency. The first phenomenon is associated with the collapse of polymers in two miscible good solvents, while the later is associated with the swelling of polymers in poor solvent mixtures. Furthermore, we will discuss when the standard Flory-Huggins type mean-field polymer theory can (or can not) be applied to understand these complex solution properties. We will also point towards future directions - how smart polymer properties can be used for the design principles of advanced functional materials. 


\section{Contents}

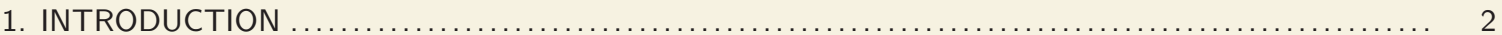

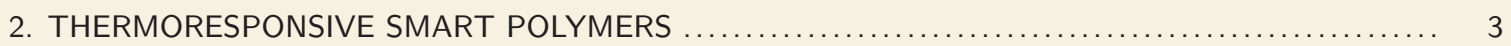

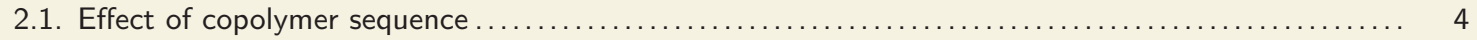

3. CO-NON-SOLVENCY: POLYMER COLLAPSE IN MISCIBLE GOOD SOLVENTS $\ldots \ldots \ldots \ldots \ldots \ldots \ldots$.

3.1. Multiscale simulations complex mixtures and polymer in mixed solvents $\ldots \ldots \ldots \ldots \ldots \ldots \ldots \ldots \ldots \ldots$

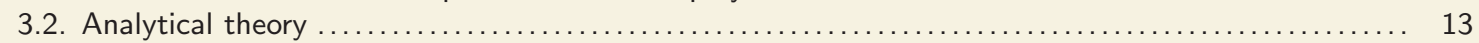

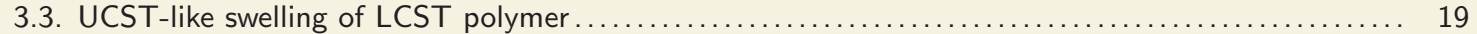

4. CO-SOLVENCY: POLYMER SWELLING IN MISCIBLE POOR SOLVENTS $\ldots \ldots \ldots \ldots \ldots \ldots \ldots \ldots \ldots 20$

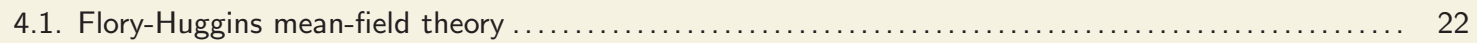

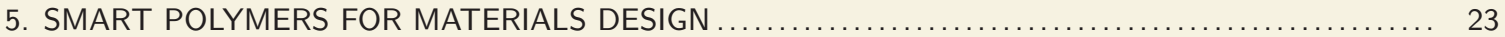

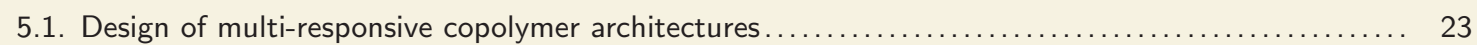

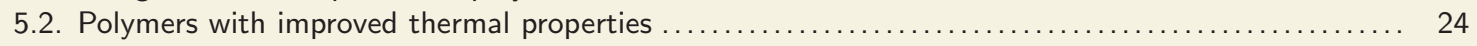

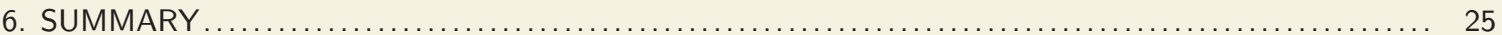

\section{INTRODUCTION}

Soft, smart and small are three keywords that are essential in designing multi-responsive materials for advanced functional applications (1, 2, 3, 4, 5, 6). A material is referred to as smart responsive when a slight change in external stimulus can drastically alter its structure, function or stability. These stimuli can be temperature $(7,8,9,10,11,12,13,14)$, ionic strength $(13,15,16,17)$, cosolvent composition $(18,19,20,21,22,23,24,25,26,27,28$, $29,30,31,32,33,34,35,36,37)$, light $(37,38,39,40,41,42,43)$, and mechanical stress $(44,45,46)$, to name a few. Furthermore, when relevant energy scale in systems is of the order of the thermal energy $k_{\mathrm{B}} T$, the materials are classified as soft matter and thus are dictated by large conformational and compositional fluctuations. Because of this strong fluctuations, entropy (generic physical concepts and scaling laws) becomes as important as energy (molecular level chemical details). Therefore, establishing a delicate balance between entropy and energy is at the heart of understanding soft matter properties.

Polymers are one class of soft materials that are of high importance as they provide suitable platform to tune materials properties, while still having rather simple materials processing. For example, establishing the microscopic understanding of the solvation behavior of smart polymeric materials, such as hydrogels, microgels, and/or composite networks in single or multi-component solvents, are of tremendous technological interests. This ranges from organic semiconductors (47), photonic band gap materials $(48,49,50,51)$, self healing networks $(52,53,54)$, tuning of thermal conductivity of thermoplastic materials $(55,56)$, and bio-medical applications $(57,58,59,60,61,62,63)$, to name a few.

In this review, we highlight recent developments in the field of smart responsive polymers and their connections to the design of smart materials. We will discuss recent experimental findings and show how complementary molecular simulation data, together with theoretical arguments, can shed light to better understand smart polymer behavior in aqueous and aqueous-cosolvent mixtures. In this context, it is important to note that polymer properties are inherently multi-scale in nature, where delicate local interaction details play a key role in describing the large scale conformational properties. We will, therefore, emphasize the need of the multi-scale modeling to arrive at a comprehensive view of the existing experimental findings. We will also discuss open questions in this field. 


\section{THERMORESPONSIVE SMART POLYMERS}

Most commonly known smart polymers are those that swiftly change their conformation upon varying temperature $T$, thus also known as thermoresponsive smart polymers. Here, $T$ responsiveness can either be classified as lower critical solution (LCST) or upper critical solution (UCST) behavior. In the case of LCST, monomer-solvent interactions confer an expanded polymer structure at low $T$ values. When $T$ is increased above a certain critical value $T_{c}$, monomer-solvent interaction becomes significantly weaker and thus solvent molecules get expelled from near the polymer backbone. In this process, the energy-entropy balance is such that the translational entropy of the released solvent molecules wins, i.e., solvent translational entropy becomes larger than the polymer conformational entropy loss upon collapse. Therefore, a chain collapses in solution. Because a chain collapses upon increasing $T$, LCST transition is an entropy driven process as already proposed by Flory $(64,65,66)$. In this case, $T_{c}$ is refereed to as $T_{\ell}$, the lower critical solution temperature of a polymer in a particular solvent. For microgels, $T_{c}=T_{\mathrm{VPTT}}$ with $T_{\mathrm{VPTT}}$ is referred to as volume phase transition temperature $(67,68,69)$. Typical examples of LCST systems are those that are mostly governed by hydrogen bonding between monomer and solvent molecules, where solvent (or one of the solvents) is usually water. On the other hand, an UCST polymer undergoes coil-to-globule transition upon decreasing $T$, making UCST an energy driven process.
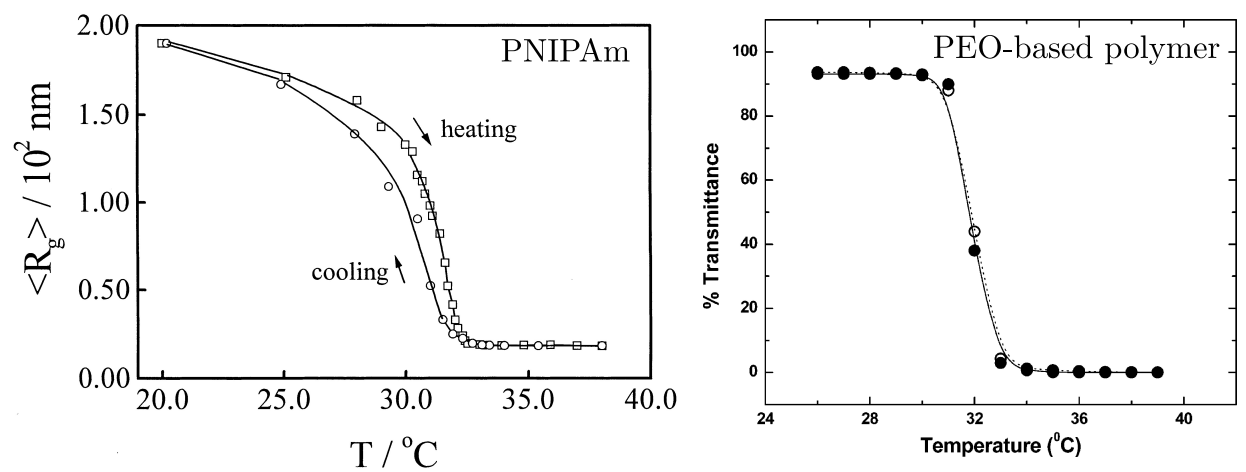

Figure 1

Left panel shows gyration radius $R_{\mathrm{g}}$ of a poly(N-isopropylcrylamide) chain in water. The transition temperature is around $32^{\circ} \mathrm{C}$ (or $305 \mathrm{~K}$ ). A hysteresis is observed between the heating and cooling cycles around this transition temperature (8). Right panel shows turbidity measurement of a semidilute solution of a poly(ethyleneoxide) based system. The transition temperature is around $32^{\circ} \mathrm{C}$ (or $305 \mathrm{~K}$ ) (13), with no hysteresis is observed around transition temperature. Figures are taken from American Chemical Society.

In a standard LCST collapse, starting from a good solvent condition (for $T<T_{\ell}$ ) increasing effective attraction between monomers first brings a polymer into a $\Theta$-condition. Further increase of of monomer-monomer attraction collapses a polymer into a compact globule. This globular conformation is dictated by balancing the second virial osmotic contributions with attractive coefficient $-|\mathcal{V}|$ and three body repulsions, were $\mathcal{V}$ is the monomer excluded volume. Furthermoremore, the $\Theta$-collapse is a second-order phase behavior, with the critical point (or the $\Theta$-point) characterized by large diverging fluctuations. Moreover, in some cases a hysteresis is also observed near $T_{\ell}$, indicating a first-order like transition and 
thus is represented by a bimodal distribution in the interaction energy $(70,71)$. It should also be mentioned that a hysteresis is usually visible for polymers with short side groups, such as the poly(N-isopropylacrylamide) (PNIPAm) $(7,8)$ and poly(N-n-propylacrylamide) (PNNIPAm) $(72,73)$. On the other hand, linear chains (such as PEO or PEO based systems) do not shown any hysteresis, see the right panel of Figure 1 for more details (13). Therefore, the specific correlation between the monomer-level structural packing and firstor a second-order like transitions for LCST systems is still not entirely understood.

For a given chemical structure of monomer species in a homopolymer chain, $T_{\ell}$ is rather well defined. In this context, an atactic PNIPAm chain has a $T_{\ell} \sim 305 \mathrm{~K}(7)$, which can, however, be tuned by slight change in monomer level chemistry. For example, by a slight increase in hydrophobicity, as seen by changing isopropyl group to n-propyl, increases hydrophobicity leading to a reduced $T_{\ell} \sim 297 \mathrm{~K}(72,73)$. On the other hand, adding an extra methyl group to the backbone alkane chain of PNIPAm, as in the case of poly(N-isopropylmethacrylamide) (PNIPMAm) (74), $T_{\ell}$ increases to $\sim 313 \mathrm{~K}$ even when an additional methyl group is expected to increase hydrophobicity. This indicates that a slight change in the monomeric structures can unexpectedly change the polymer properties and thus lead to noticeably different $T_{\ell}$.

$T_{\ell}$ can also be tuned by changing the tacticity of a homopolymer chain. For example, going from a chain with $100 \%$ meso diads (isotactic chain) to $0 \%$ meso diads or $100 \%$ racemo diads (syndiotactic chain), $T_{\ell}$ follows the trend $T_{\ell}^{\text {isotactic }}<T_{\ell}^{\text {atactic }}<T_{\ell}^{\text {syndiotactic }}$ with 5050 combination of meso and racemo diads approximately corresponding to an atactic chain $(75,76,77)$. Here, the stiffness of a chain, as measured in terms of the Kuhn length $\ell_{k}$, follows the trend $\ell_{k}^{\text {isotactic }}<\ell_{k}^{\text {atactic }}<\ell_{k}^{\text {syndiotactic }}(77)$. In this context, it should also be noted that following simple entropic arguments, the stiffer the chain the lesser its solubility. This will then corresponds to decreasing LCST with increasing $\ell_{k}$. Here, however, we observe an opposite trend that is attributed to the different solvation structure around the side groups and are more exposed when a chain has syndiotactic tacticity (or altering side groups). Another possible route to tune $T_{\ell}$ is by copolymerization. This is discussed in the following section.

\subsection{Effect of copolymer sequence}

A more flexible tuning of $T_{\ell}$ can be achieved by introducing hydrophilic (or hydrophobic) monomers along the native polymer backbone, where the more hydrophilic (or hydrophobic) is described in comparison to the native homopolymer chain. More specifically, introducing hydrophilic units usually increases $T_{\ell}$, while hydrophobic units decrease $T_{\ell}$. An example includes copolymer sequence poly(NIPAm-co-Am) consisting of two monomers: acrylamide $(\mathrm{Am})$ and N-isopropylcrylamide (NIPAm) $(78,79)$. It should be noted that Am is more hydrophilic than NIPAm, where no $T_{\ell}$ is reported within the range of $273-350 \mathrm{~K}$ in pure water. Therefore, as expected, increasing mole fraction of Am $x_{a}$ along a PNIPAm backbone also increases $T_{\ell}$, see a comparative simulation and experimental plot in Figure $2(77,78,79)$. Moreover, it can be seen that the increase in $T_{\ell}$ is difficult to predict and nonlinear with increasing $x_{a}$. Therefore, it is desirable to have a more predictive and tunable polymer sequence. In this context, copolymer sequences consisting of hydrophobic (methylene) and hydrophilic (ethylene-oxide) monomer units (see Figure 3(a)) was recently synthesized (13), which show highly predictive thermal responsivess. Added advantage of these systems is that they are acetal linked, making them $\mathrm{pH}$ degradable (13). In this context note 


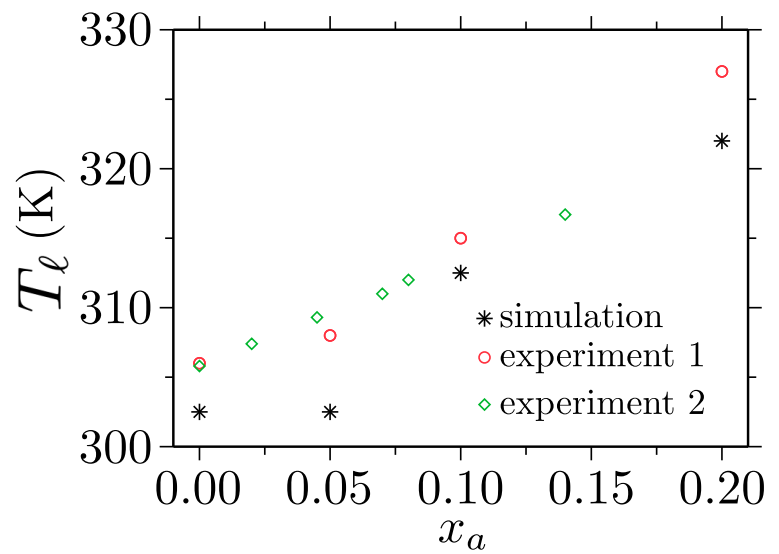

Figure 2

Lower critical solution temperature $T_{\ell}$ of a random copolymer of poly(N-isopropylacrylamide) and poly(N-isopropylacrylamide) as a function of acrylamide mole fraction $x_{a}(77)$. Figure is taken from American Institute of Physics.

that carbon-carbon bond strength is of the order of $80 k_{\mathrm{B}} T$ and, therefore, these bonds live for ever under unperturbed circumstances, leading to severe environmental problems. Acetal linkage made these copolymers biodegradable and highly suitable bio-compatible polymers. Polyacetal, as termed in Ref. (13), increasing the fractions of hydrophobic (represented by $n_{i}$ ) or hydrophilic (represented by $m_{i}$ ) units (see Figure 3(a)), linearly changes $T_{\ell}$ of a copolymer chain. Because of this linear behavior, which was also observed in a generic molecular simulation study (80), these copolymer sequences provide a rather flexible molecular toolbox for desired applications. Moreover, these systems also show severe
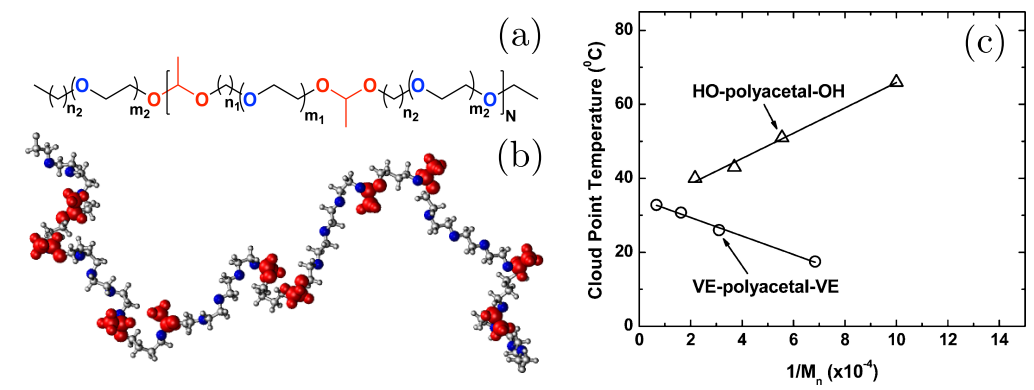

Figure 3

Parts (a) and (b) show chemical structure and simulation snapshot of a polyacetal chain (81). Here the hydrophobic methylene units (represented by $n_{1}$ and $n_{2}$ ) and hydrophilic ethylene oxide units (represented by $m_{1}$ and $m_{2}$ ) are tuned to obtain different amphiphilic sequences. Part (c) presents a Fox-Flory relationship showing transition temperature with inverse of the molecular weight $M_{n}$ for a given copolymer sequence(13). The parts (a) and (b) is reproduced from American Institute of Physics and part (c) is taken from American Chemical Society.

chain length effects. As shown in Figure 3(c), a molecular weight $M_{n}$ of above $10^{4} \mathrm{~g} / \mathrm{mol}$ is required to obtain a well converged cloud point temperature $T_{\mathrm{cp}}^{\infty}$. Moreover, depending of 
hydrophilic (-OH) or hydrophobic (vinyl ester -VE) termination, $T_{\mathrm{cp}}$ shows different slopes with $M_{n}$, with both following Flory-Fox relationship $(64,65,66)$,

$$
T_{\mathrm{cp}}=T_{\mathrm{cp}}^{\infty}-\frac{\text { const. }}{M_{n}} .
$$

Furthermore, as indicated by Figure 3(c) specific chemical details matter for rather short chain lengths (or oligomeric units) because of their end termination, this indicates that the global polymer behavior in the asymptotic limit is independent of the end termination. Therefore, while the copolymer structure is given by the statistical distribution of polymer segments, the global polymer conformation is well described by scaling laws.

2.1.1. Systematic structural coarse-grained model. While polyacetal based systems are highly important for smart materials design, to further increase their usefulness will require a rather large set of polymer architectures with predictable behavior. This, however, in experiments is not trivial at all. Therefore, coarse-grained (CG) simulation models have been also developed to study these systems (81). In this context, a linear dependence of $T_{\mathrm{cp}}$ with polymer sequences, as seen from the experiments (13), indicates that the effect of one monomer type is independent of the effects induced by the other monomer type. Therefore, a systematic CG model was developed at the segment (or monomer) level. A simple mapping scheme is shown in Figure 4(a), with two corresponding copolymer sequences in Figures 4(b) and (c). For the derivation of CG model a combination of two

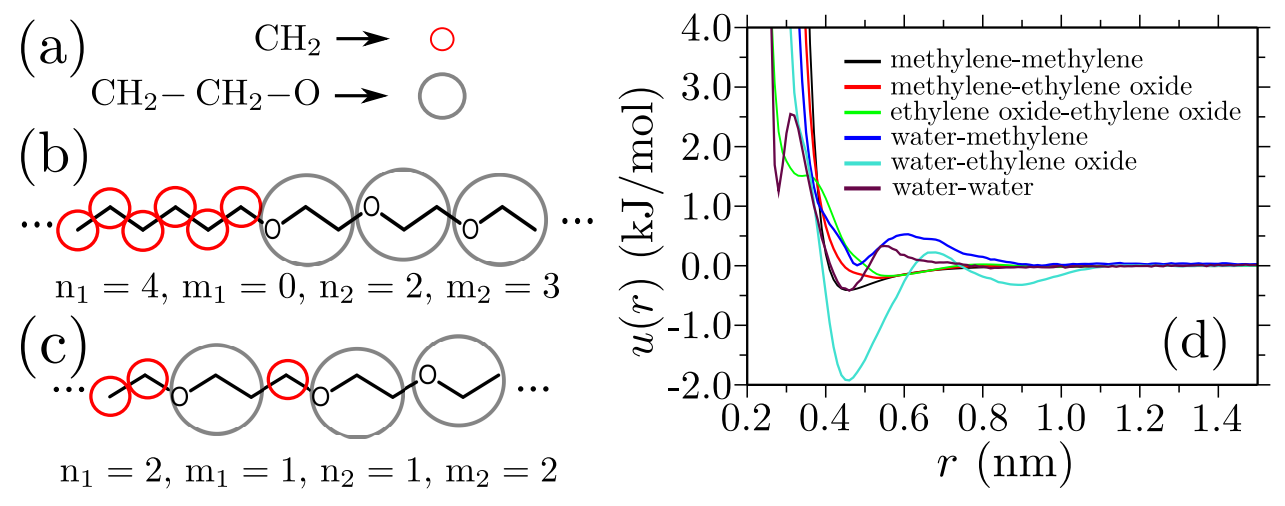

Figure 4

Parts (a-c) show a mapping scheme of methylene and ethylene oxide monomers and two different copolymer sequences, namely $n_{1}=4, m_{1}=0, n_{2}=2$, and $m_{2}=3$ and $n_{1}=2, m_{1}=1, n_{2}=1$, and $m_{2}=2$, respectively (81). Part (d) shows the pairwise coarse-grained potentials. Figure is taken from American Institute of Physics.

structure-based techniques for solutions were used $(82,83)$ : namely the iterative Boltzmann inversion (IBI) (84) and the cumulative iterative Boltzmann inversion $(\mathcal{C}-\mathrm{IBI})(85)$. In a nutshell, the IBI procedure starts from an initial guess of the interaction potential of the CG model $\mathrm{V}_{0}(r)=-k_{\mathrm{B}} T \ln \left[\mathrm{g}_{i j}^{\text {target }}(r)\right]$. Here $\mathrm{g}_{i j}^{\text {target }}(r)$ is the pair distribution function between different solvent components obtained from the reference all atom simulation. Then the potentials are updated over several iterations $n$ using the protocol, $\mathrm{V}_{n}^{\mathrm{IBI}}(r)=$ $\mathrm{V}_{n-1}^{\mathrm{IBI}}(r)+k_{\mathrm{B}} T \ln \left[\mathrm{g}_{i j}^{n-1}(r) / \mathrm{g}_{i j}^{\text {target }}(r)\right]$. Moreover, in the IBI protocol, solution component fluctuations that are related to the tail of $\mathrm{g}_{i j}(r)$ sometimes need fine tuning, especially when 
dealing with multi-component systems. For this purpose, the $\mathcal{C}$-IBI might serve as a possible candidate. In $\mathcal{C}-\mathrm{IBI}$, the initial guess of potential is taken from IBI, i.e., $\mathrm{V}_{n}^{\mathrm{IBI}}(r)$, which is then updated with a protocol, $\mathrm{V}_{n+1}^{\mathcal{C}-\mathrm{IBI}}(r)=\mathrm{V}_{n}^{\mathcal{C}-\mathrm{IBI}}(r)+k_{\mathrm{B}} T \ln \left[\mathcal{C}_{i j}^{n}(r) / \mathcal{C}_{i j}^{\text {target }}(r)\right]$, with cumulative integral $\mathcal{C}_{i j}^{n}(r)=4 \pi \int_{0}^{r} \mathrm{~g}_{i j}^{n}\left(r^{\prime}\right) r^{\prime 2} d r^{\prime}$. by this a single set of CG potentials (see Figure $4(\mathrm{~d})$ ), obtained from the monomer level simulations of individual monomer species, could explain a broad range of copolymer sequence (81) synthesized experimentally (13), see Table 1. This makes the CG model obtained from a structure based CG method sequence transferable (81). These CG simulations were performed for the polymer chains $M_{n} \simeq 10^{4}$ $\mathrm{g} / \mathrm{mol}$.

Table 1 Copolymer conformations with different sequences $n_{1}, m_{1}, n_{2}$, and $m_{2}$. In the last two columns, a comparison of polymer conformation from experiments and CG simulations are shown. Note- experiments were performed around the semidilute regime and simulations data are shown for single chain limit. Therefore, turbid solution corresponds to collapsed object and clear solution shows good solubility, i.e., expanded chain conformation (81). Table is taken from American Institute of Physics.

\begin{tabular}{|l|l|l|l|l|l|}
\hline$n_{1}$ & $m_{1}$ & $n_{2}$ & $m_{2}$ & Experiment (13) & Simulation (81) \\
\hline \hline 4 & 0 & 2 & 1 & Turbid & Globule \\
\hline 4 & 0 & 2 & 2 & Turbid & Globule \\
\hline 4 & 0 & 2 & 3 & Turbid & Globule \\
\hline \hline 2 & 1 & 2 & 1 & Turbid & Globule \\
\hline 2 & 1 & 2 & 2 & Turbid & Globule \\
\hline 2 & 1 & 2 & 3 & Clear & Lamellar \\
\hline \hline 2 & 2 & 2 & 1 & Clear & Globule \\
\hline 2 & 2 & 2 & 2 & Clear & Expanded \\
\hline 2 & 2 & 2 & 3 & Clear & Expanded \\
\hline
\end{tabular}

Another class of bio-compatible LCST polymers is the elastin-like polypeptides (ELP). ELPs are usually a five amino acid based copolymer consisting of a sequence Val-Pro-Gly$\mathrm{X}$-Gly. Here $\mathrm{X}$ can be any amino acid except proline $(9,86,87)$. These polymers often display a coil-to-globule transition with increasing temperatures, thus exhibiting an LCST transition. These synthetic polypeptides are not only fundamentally interesting, but offer a broad range of bio-technological applications.

2.1.1.1. Extension of coarse-grained model for brush polymers. Simple CG model as the one discussed above can also effectively capture the solution behavior of other polymer architectures (such as the bottle brush polymers) consisting of alkane backbone and ethylene oxide side chains (88). In this context, it was experimentally shown that- while single bottle brush polymer consisting of ethylene oxide side chain and alkane backbone is well soluble in aqueous solutions, around semi-dilute concentration these polymers form large aggregates. As elucidated by the all-atom simulations (see simulation snapshot in the left panel of Figure 5), these bottle brush systems can self assemble in their semi-dilute solution. These aggregates are due to the interdigitation of ethylene oxide side chains that originate due to attraction between hydrophobic $-\mathrm{CH}_{2}$ patches within the side chains. More interestingly, the CG potential obtained in Figure 4(d) can well reproduce the agregation behavior of the bottle brush polymer, see right panel of Figure 5. Note that in the CG model molecular weights of polymer chains are exactly same as in the experimental system (88). 
all-atom

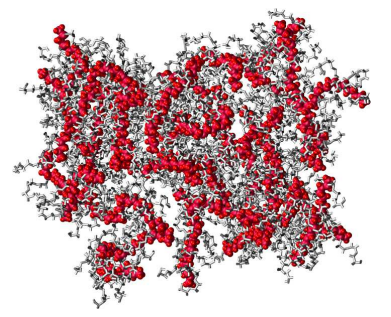

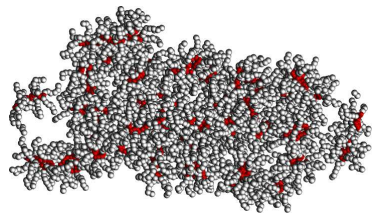

Figure 5

Simulation snapshot showing aggregation of bottle brush polymer consisting of alkane backbone and ethylene oxide side chains (88). A comparison of all-atom and coarse-grained simulations results are shown. Note- the molecular weight $M_{n}$ for all-atom simulations were half the $M_{n}$ from experimental synthesis. For CG simulation, $M_{n}$ is same as experimental polymers.

2.1.1.2. Temperature transferability of coarse-grained model. The CG model described above is transferable with changing sequences, they are not transferable with changing $T$. For example, structure based CG methods are dependent on pair-wise structure that inherently depends on temperature, thus making structure-based CG models state point dependent. In this context, however, CG model for a PNIPAm chain in bundled water (four water molecules clustered into one CG bead) was developed (89) based on the CG force field $(90,91)$. This work presents results for two different temperatures, where two different sets of pair-wise $\mathrm{CG}$ potentials were used to account for the temperature effect. Furthermore, when a polymer goes from coil-to-globule transition, it is dictated by a delicate balance between entropy and energy near the transition point that originate from the three-body effect. It should still be mentioned that the collapsed transition itself does not need three body effects. Moreover, three-body effects guarantees a finite density in the collapsed state. In general CG energies are free energies when compared to the all-atom systems. Thus are linked to a thermodynamic state point and one can not expect temperature transferability. However, if a CG model can properly account for this delicate entropy-energy balance, as in the case of azobenzene (92), it is also expected that the underlying CG model may also be able to give temperature transferability.

\section{CO-NON-SOLVENCY: POLYMER COLLAPSE IN MISCIBLE GOOD SOLVENTS}

So far we have discussed polymer properties in single component solvents and their coil-toglobule transition with change in temperature. However, the conformational behavior of a polymer also can be greatly influenced by the presence of small cosolvent molecules within the solvation volume of a polymer in solution, because of the competitive interaction of solvent and cosolvent with polymer. This is because cosolvents can often drastically alter the solvation structure and thus the solvation free energy of a polymer $(18,19,20,24,26)$. For example, starting from an expanded chain of PNIPAm in pure water below its $T_{\ell}$, addition of cosolvent (especially small alcohols and other organic solvents) first decreases $T_{\ell}$ and then $T_{\ell}$ eventually sharply increases when alcohol content increases beyond a certain concentration $(18,19)$. In Figure 6 a representative phase diagram of PNIPAm in aqueous methanol 


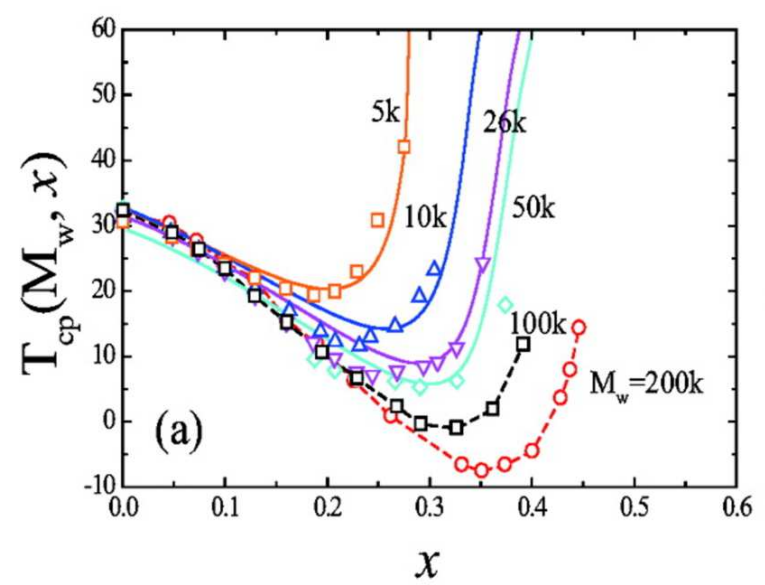

Figure 6

Lower critical solution temperature of poly(N-isopropylacrylamide) in aqueous methanol mixture for different methanol concentrations. Data is shown for different polymer molecular weights. The higher the molecular weight the broader the window of collapse. The region above the phase lines shows phase separated (two-phase) system, while the miscible region is below the phase line (26). Figure is taken from American Chemical Society.

mixture is shown. This phase diagram clearly indicates that, for a given temperature (lets say the room temperature $298 \mathrm{~K}$ ), increasing molar cosolvent concentration $x_{c}$ (in this case alcohol) first causes the chain to collapse and then to expand again, when $x_{c}$ is increased above a critical value. This coil-globule-coil transition is refered to as co-non-solvency. Interestingly, even when the co-non-solvency of PNIPAm was reported in 1991 (18, 19), the term co-non-solvency was first already coined in 1978 to describe the solvation of polystyrene in a mixture of cyclohexane-DMF solution (93). It should also be highlighted that water and short alcohols (such as methanol and ethanol) are well miscible over the full water-alcohol mixing ratios, while larger alcohols (such as propanol and butanol) becomes lesser-and-lesser soluble in water. Here, if the bulk solvent-cosolvent solution was phase separated, it would be quite obvious to explain the phenomenon of co-non-solvency. For example, in a phase separated binary mixture a polymer would prefer to sit in the interfacial region between two solvents because of the reduced interfacial tension and increasing polymer concentration would lead to polymer collapse. In this case, however, a single chain would not show a coil-to-globule transition (32). On the other hand, if both solvents were strongly attractive compared to monomer-solvent or monomer-cosolvent interactions, one would expect to have a polymer falling out of solution driving the phase separation $(94,95)$. Here, however, it should be mentioned that the common solvent mixtures, where co-non-solvency is observed, are fairly miscible (but not perfectly miscible) as in the case of water and alcohol. Data that somewhat speak in this favor is the density variation of aqueous alcohol solution with changing relative mixing ratios, i.e., total number density reduces from the mean-field value with a minima around 50-50 mixing. This is because of rather weak steric repulsion between two components (96).

What causes co-non-solvency in fairly miscible binary mixtures? More specifically, to understand factors that cause a decrease in $T_{\ell}$ with increasing cosolvent concentration and 
not that the original $T$ driven of collapse of a chain in water is entropy driven. In this context, to reveal the microscopic origin of this puzzling behavior, extensive studies have been performed using experiments, theory and computer simulations. In view of these studies, three main pictures were proposed to understand the microscopic origin of co-non-solvency: based on solvent-cosolvent interactions $(18,32,94,95)$, cooperative polymer-solvent and polymer-cosolvent hydrogen bonding $(24,26)$, and preferential polymer-cosolvent binding $(28,29,30)$. Therefore, we proceed here with highlighting results from the molecular simulations.

\subsection{Multiscale simulations complex mixtures and polymer in mixed solvents}

As mentioned earlier, polymer properties are governed by a rather delicate energy (details of the chemical structure) and entropy (generic, universal scaling laws, critical phenomena) interplay. This connection is at heart of the understanding of many biological as well as synthetic materials and processes. At the same time it is very difficult to address the questions to understand details within conventional experimental and mid sized canonical (NVT) or isobaric (NpT) simulation setups, where $\mathrm{N}$ is the number of particles in the simulation box, $\mathrm{V}$ is volume of the system, $\mathrm{T}$ is the temperature and $\mathrm{p}$ is the pressure. Therefore, there is a need to address these problems within multiscale simulation approaches, where local microscopic interaction details (where local is referred to the range of correlation length that is typically less than $2.0 \mathrm{~nm}$ in these water soluble systems) are coupled in equilibrium with a large solvent bath allowing for global conformational fluctuations. For example, polymer properties in mixed solvents typically are dictated by large conformational and (co-)solvent compositional fluctuations. Computer simulations in canonical ensemble usually, however, suffer from system size effects $(97,98)$. This is partially due to the fact that the local agregation of one of the solvent components at one place of simulation box leads to the depletion of the same species at other region within the simulation box. This disturbs the solvent equilibrium and thus leads to wrong fluctuations.

A quantity that gives the direct measure of the fluctuation within the simulation domain is,

$$
\mathrm{G}_{i j}=4 \pi \int_{0}^{\infty}\left[\mathrm{g}_{i j}^{\mu \mathrm{VT}}(r)-1\right] r^{2} d r=\mathrm{V}\left[\frac{\left\langle N_{i} N_{j}\right\rangle-\left\langle N_{i}\right\rangle\left\langle N_{j}\right\rangle}{\left\langle N_{i}\right\rangle\left\langle N_{j}\right\rangle}-\frac{\delta_{i j}}{\left\langle N_{j}\right\rangle}\right]
$$

where thermal averages are denoted by brackets $\langle\cdot\rangle$. V is the volume, $N_{i}$ is the number of particles of species $i, \delta_{i j}$ is the Kronecker delta, $\mathrm{g}_{i j}^{\mu \mathrm{VT}}(r)$ is the radial distribution function in the $\mu \mathrm{VT}$ ensemble $(30,98,99,100,101,102)$. Here, $\mathrm{G}_{i j}$ is refereed differently in different communities. In bio-physical community $\mathrm{G}_{i j}$ is known as the Kirkwood-Buff integrals (KBI) (99) and in statistical physics $\mathrm{G}_{i j} / 4 \pi$ is known as the Mayer's function. $-\mathrm{G}_{i j} / 2$ also gives direct measure of the second virial coefficient $\beta_{2}$ (or the excluded volume $\mathcal{V}$ ), a highly useful quantity to describe polymer conformation $(6,29)$. Furthermore, $\mathrm{G}_{i j}$ is a local quantity that can be used as a measure of the affinity between solution components $i$ and $j$. A positive (or negative) value of $\mathrm{G}_{i j}$ refers to excess (or depletion) of component $j$ around component $i$. $\mathrm{G}_{i j}$ can also be used to calculate solvation thermodynamics of multi-component complex fluids.

Following Eq. $2 \mathrm{G}_{i j}$ should be calculated in a grand canonical ensemble, while in a closed boundary setup $\mathrm{G}_{i j}$ can only be estimated at $r \rightarrow \infty$ (99). A typical cumulative $\mathrm{G}_{i j}$ is shown in Figure 7(a). It can be seen that for a small system size (consisting of $6 \times 10^{3}$ molecules) $\mathrm{G}_{i j}(r)$ suffers from severe system size effects and it is impossible to get 

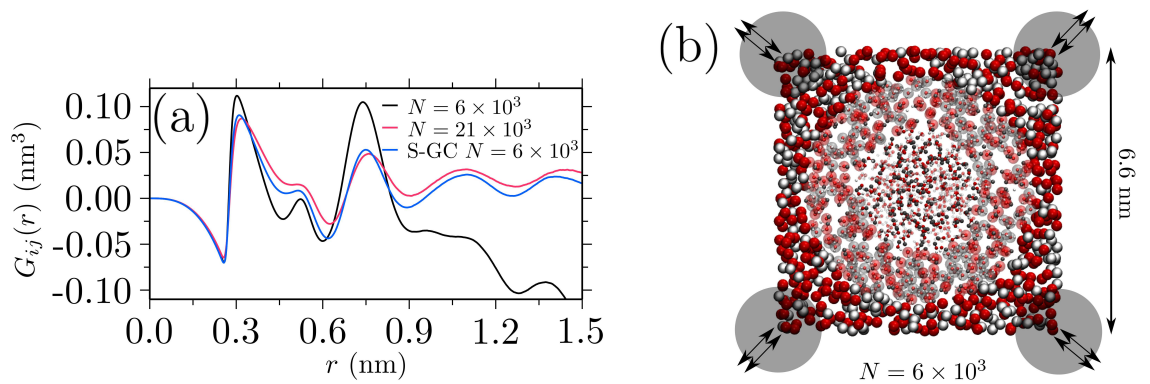

Figure 7

Part (a) shows Kirkwood-Buff integrals (KBI) of aqueous methanol mixture for methanol mole fraction of $x_{c}=0.90$. Results are shown for two different methods: all-atom simulations and semi-grand canonical (S-GC) molecular dynamics. Also for two different system sizes. Part (b) shows a schematic representation of the S-GC scheme with periodic boundary condition.

Conventional adaptive resolution scheme (AdResS) is used (104), where a small AA region is coupled to a much smaller S-GC reservoir. The particle exchange is performed at the corners keeping the constant chemical potential. The size of the AA is similar to correlation length of the molecular liquid. In the AA description: oxygen is shown in red, hydrogen in silver and the united atom $\mathrm{CH}_{3}$ is shown in steel. Coarse-grained representation: the red and silver beads represent methanol and water, respectively (30). The figures are taken from American Chemical Society.

any reasonable converged value for $\mathrm{G}_{i j}$, which can, however, be estimated from a 21000 molecule system (see red curve) (30). This clearly demonstrates that a large system size is required to maintain solvent equilibrium and to avoid severe system size effects. In this context, because the original KB theory is derived in a $\mu \mathrm{VT}$ ensemble, it might be better to estimate these quantities within a grand canonical environment.

Implementation of grand canonical environment in molecular simulation requires to introduce particle insertion and deletion moves. Moreover, in a molecular liquids, particle insertion suffers from poor acceptance rates because of significant particle overlap (103). Therefore, an alternative is to use a semi-grand canonical protocol, where particle identities are exchanged satisfying a Metropolis particle exchange criterion. For example, in a binary complex mixture, based on the chemical potential (or relative composition) of a particular solution, paticle identity is switched between one species and other. Even this also suffers from poor acceptance rates for an all atom simulation. If instead molecules are represented by CG spheres, their exchange can be much easier.

Semi-grand canonical molecular dynamics (S-GC) combined with an adaptive resolution scheme (AdResS) (104) has been used for this purpose. In AdResS, a high resolution all atom simulation domain is coupled with a low resolution CG reservoir with periodic boundary conditions. Particle exchange is introduced in the CG reservoir. A schematic of the S-GC scheme is shown in Figure 7(b). Particle exchange was performed at eight corners of the simulation domain (represented by grey spheres). Using S-GC scheme nicely converged KBI can be obtained (see blue curve in Figure 7(a)). Therefore, S-GC scheme guarantees solvent equilibrium and thus can be used to study complex conformational transition of large macromolecules. There are also other methods to compute thermodynamic quantities from particle fluctuations from open boundary molecular simulations $(99,98,30,100,101,102)$

Figure 8 shows gyration radius $R_{\mathrm{g}}$ as a function of methanol mole fraction $x_{c}$. Data is shown from S-GC simulations (part (a)) and a master curve (part(b)) (30). Furthermore, 
Figure 8(b) also shows data from generic simulations, experimental data, and analytical theory. Note that detailed theory will be discussed in a later section. Here generic simulations are based on only Lennard Jones (LJ) interactions between different solution components that are tuned to reproduce correct solvation free energy known from all-atom simulations. Furthermore, for an atactic PNIPAm chain one has $\ell_{p} \sim 2.5-3$ monomers or $\ell_{p} \sim 1 \mathrm{~nm}$. In our generic simulations, one bead (of size $1 \sigma$ ) corresponds to 2.5-3 NIPAm monomers. This leads to a mapping of $1 \sigma \sim 1 \mathrm{~nm}(105)$.
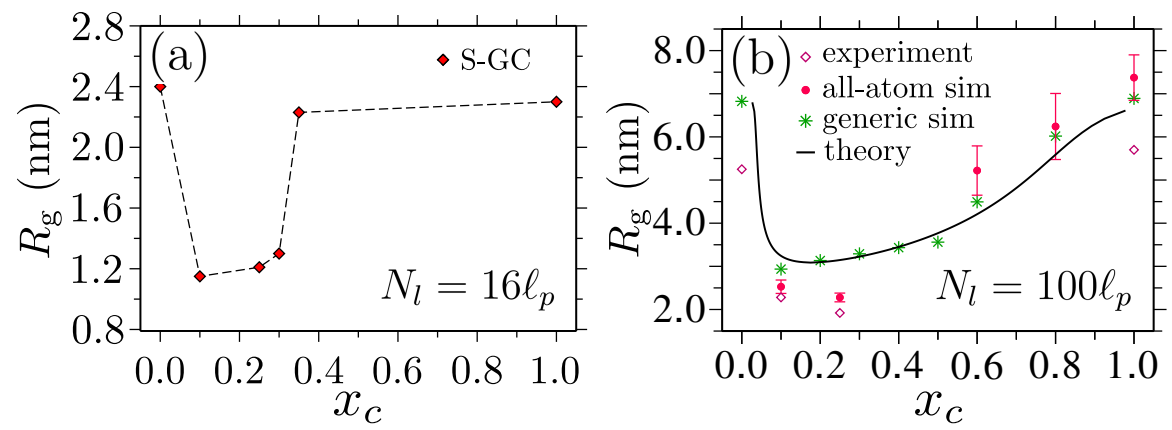

Figure 8

Gyration radius $R_{\mathrm{g}}$ as a function of cosolvent mole fraction $x_{c}$. Part(a) shows results from semi-grand canonical molecular dynamics (S-GC) for a chain length of $N_{l} \sim 16 \ell_{p}$, with $\ell_{p}$ being the persistence length (30). Part (b) shows a master curve of all-atom simulations, generic simulations, and experimental measurements for $N_{l} \sim 100 \ell_{p}$. For comparison, analytical plot is also included for $N_{l}=100 \ell_{p}$ (105). Figures are taken from American Chemical Society (left panel) and Royal Society of Chemistry (right column).

It can be appreciated that the coil-globule-coil behavior of polymers in miscible good solvents is observed irrespective of simulation model or method. Another interesting observation appears from Figure 8 is that - the longer the chain, the broader the window of collapse as also observed earlier (26) Here, however, the goal of simulation is not only to reproduce experiments, rather is also to give direct molecular level description of the mechanism governing properties of a physical system.

3.1.1. Excess coordination and chemical potential. Whenever a polymeric system undergoes a conformational transition, it is associated with the modification of solvent structure within the solvation volume of the polymer. In Figure 9(a) the relative mole fraction of cosolvent $x_{c}^{*}$ as a function of radial distance $r$ from the polymer backbone with respect to the bulk solution cosolvent mole fraction $x_{c}=0.1$ is shown $(69,106)$. In a nutshell, $x_{c}^{*}>1$ shows an excess of cosolvent, while $x_{c}^{*}=1$ shows the bulk solution mole fraction. It can be appreciated that within the first solvation shell (i.e., $r<0.6 \mathrm{~nm}$ ) there is almost three fold increase in cosolvent composition, which is a result of cosolvent molecules replacing solvent because of their preferential binding with the polymer. To test this observation of preferential cosolvent-monomer interactions proton NMR measurements are shown in Figure 9(b) (105). During a measurement spanning over about 16 days, composition of methanol was monitored in the lower panel of the NMR tube (see the inset in Figure 9(b)). It can be seen that there is a reduction of about $3 \%$ alcohol in the lower panel of the tube, indicating that the concentrated polymer solution in the top panel attracts a significant amount 

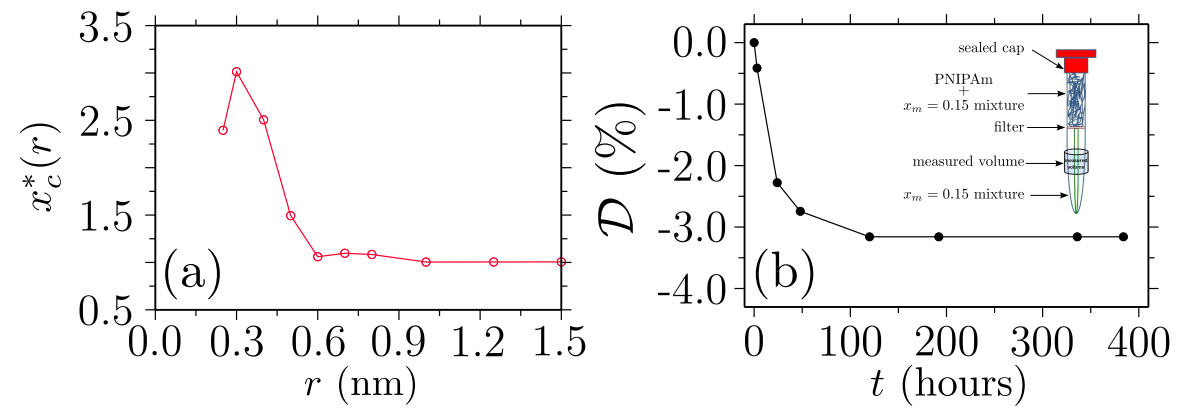

Figure 9

Part (a) shows excess cosolvent mole fraction $x_{c}^{*}$ as a function of radial distance $r$ from the polymer backbone (106). Part (b) displays proton nuclear magnetic resonance (NMR) data showing solvent uptake by the upper panel of the NMR tube (inset) containing polymer (105). Figure is taken from Royal Society of Chemistry.

of alcohol molecules. It should also be mentioned- even when $3 \%$ seem to be a rather small number, this is still significant considering that the volume of the lower panel in the NMR experiments is much larger than the top panel. Additionally, plateau in $\mathcal{D}$ after four to five days also indicates that there is no solvent evaporation within the air-sealed NMR tube. It should be highlighted that earlier works also showed that the preferential cosolventmonomer drives co-non-solvency $(107,108)$. A more recent work, however, also emphasized that the preferential cosolvent-monomer binding is not a prerequisite for co-non-solvency (109)

As it turned out the preferential binding of one of the solvents to the polymer is driving the conformational transition of a polymer in mixed good solvents. This is in particularly important because if both solvents like polymer equally then only weak traces of the nonideal mixing of the solvents are left, which are not sufficient to drive segregation. Only when one of the (co)solvents like polymer more than the other, then one could expect coilglobule-coil transition observed in experiments. Furthermore, this preferential binding is connected to very interesting thermodynamic properties (30). For example, in Figure 10 chemical potential of a polymer $\mu_{p}$ as a function of $x_{c}$ is shown. $\mu_{p}$ monotonically decreases with increasing $x_{c}$, thus indicates that the solvation of polymer is becoming better-andbetter with the increasing concentration of the better cosolvent. This is counterintuitive and puzzling, given that when a polymer goes from coil-to-globule transition it should get thermodynamically costlier to solvate the polymer, i.e. the solvent becomes a "poor" solvent. Here we see strikingly different thermodynamic behavior. Therefore, within the simple thermodynamic arguments, co-non-solvency based conformational transition would lead to low-high-low type behavior of $\mu_{p}$ and not the ever decreasing solvation free energy, as seen in Figure 10. Therefore, there is a need to understand this phenomenon within theoretical framework.

\subsection{Analytical theory}

The theoretical challenges posed by co-non-solvency were identified early on (18), as the first Flory-Huggins description of the observed collapsed was attempted. First theoretical progress was achieved later when more accurate theories for the LCST behavior of PNIPAM 


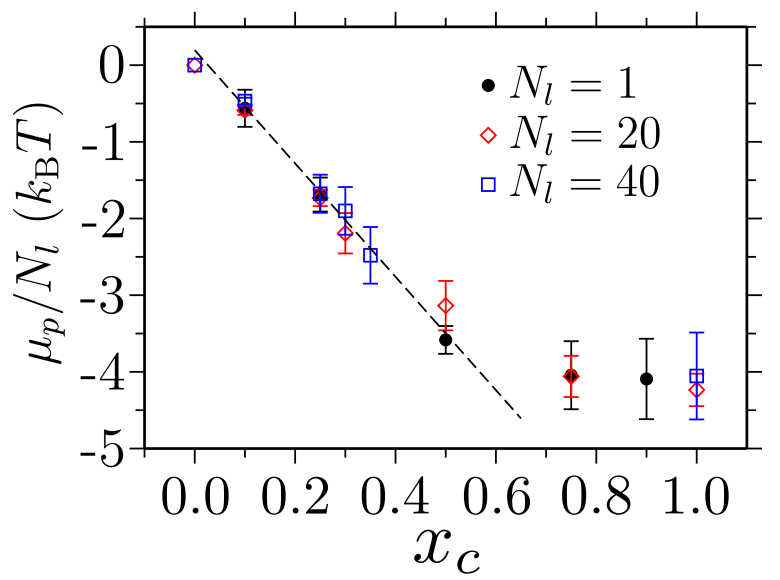

Figure 10

A master curve showing the shift in chemical potential $\mu_{p}$ per monomer as a function of cosolvent mole fraction (30). The data was obtained for a temperature of $298 \mathrm{~K}$, where $k_{\mathrm{B}} T=2.5 \mathrm{~kJ} / \mathrm{mol}$. Figure is taken from American Chemical Society.

became available $(24,26)$. However, not until recently was the generic character of this phenomena recognized and explained by a combination of analytical theory and numerical simulations $(3,110)$.

3.2.1. Flory-Huggins mean-field theory. A standard thermodynamic theory to describe polymer conformation is the mean-field theory of Flory-Huggins. In this theory, when a polymer $p$ with chain length $N_{l}$ at volume fraction $\phi_{p}$ is dissolved in a binary mixture of solvent $s$ and cosolvent $c$, the Flory-Huggins free energy $\mathcal{F}_{\mathrm{FH}}$ of polymer solutions is written as $(64,65,66)$,

$$
\begin{aligned}
\frac{\mathcal{F}_{\mathrm{FH}}}{\kappa_{\mathrm{B}} T} & =\frac{\phi_{p}}{N_{l}} \ln \phi_{p}+x_{c}\left(1-\phi_{p}\right) \ln \left[x_{c}\left(1-\phi_{p}\right)\right]+\left(1-x_{c}\right)\left(1-\phi_{p}\right) \ln \left[\left(1-x_{c}\right)\left(1-\phi_{p}\right)\right] \\
& +\chi_{p s} \phi_{p}\left(1-x_{c}\right)\left(1-\phi_{p}\right)+\chi_{p c} \phi_{p} x_{c}\left(1-\phi_{p}\right)+\chi_{s c} x_{c}\left(1-x_{c}\right)\left(1-\phi_{p}\right)^{2}
\end{aligned}
$$

Here, the first three terms represent the entropy of mixing and the last three terms deal with interactions between different components $i$ and $j$ through $\chi_{i j}$ parameters. The second order expansion of Eq. 3 gives a direct measure of the excluded volume $\mathcal{V}$ of the polymer,

$$
\mathcal{V}=1-2\left(1-x_{c}\right) \chi_{p s}-2 x_{c} \chi_{p c}+2 x_{c}\left(1-x_{c}\right) \chi_{s c},
$$

In a nutshell, under a good solvent condition for a polymer $\mathcal{V}>0$ with the scaling law for gyration radius and single chain static structure factor $S(q)$ following $R_{\mathrm{g}} \sim N_{l}^{3 / 5}$ and $S(q) \sim q^{-5 / 3}$, respectively. Increasing monomer-monomer attraction first brings a polymer into the $\Theta$-condition where long range energetic attraction gets exactly cancelled by the short range entropic repulsion. At the $\Theta$-condition $\mathcal{V}=0, R_{\mathrm{g}} \sim N_{l}^{1 / 2}$ and $S(q) \sim q^{-2}$. When the monomer-monomer attractions is even further increased, a polymer collapses into a compact globule where $\mathcal{V}<0, R_{\mathrm{g}} \sim N_{l}^{1 / 3}$ and $S(q) \sim q^{-4}(64,65,66)$.

When both solvent and cosolvent are good solvents for a polymer, $\chi_{p s}<1 / 2$ and $\chi_{p c}<1 / 2$ (18). If the bulk binary solution is perfectly miscible (i.e. $\chi_{s c}=0$ ) the first two 

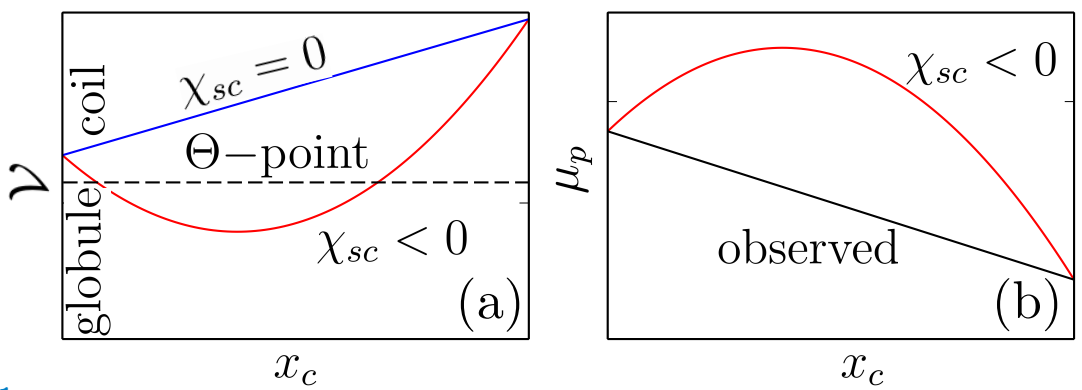

Figure 11

Parts (a) and (b) show schematic representations of the polymer excluded volume $\mathcal{V}$ and chemical potential of polymer $\mu_{p}$ as a function of cosolvent mole fraction $x_{c}$. The horizontal line (dashed black) in part (a) shows $\Theta$-point when $\mathcal{V}=0$. Figures are taken from American Institute of Physics.

terms of Eq. 4 give a linear variation of $\mathcal{V}$ with $x_{c}$, see the blue line in Figure 11(a). Only when $\chi_{s c}<0$ can $\mathcal{V}$ become negative, opening the possibility for the coil-to-globule-to-coil conformation changes typical of co-non-solvency, see the red line in Figure 11(a).

Furthermore, within the mean-field picture in Eq. 3, the shift in chemical potential of polymer $\bar{\mu}_{p}$ under infinite dilution $\phi_{p} \rightarrow 0$ can be written as,

$$
\begin{aligned}
\bar{\mu}_{p}\left(\phi_{p} \rightarrow 0\right) & =\left.\frac{\partial \mathcal{F}_{\mathrm{FH}}}{\partial \phi_{p}}\right|_{\phi_{p} \rightarrow 0} \\
& =\text { const }-x_{c} \ln x_{c}-\left(1-x_{c}\right) \ln \left(1-x_{c}\right)+\left(1-x_{c}\right) \chi_{p s}+x_{c} \chi_{p c}-2 x_{c}\left(1-x_{c}\right) \chi_{s} 5 .
\end{aligned}
$$

In Figure 11(b) a schematic representation of $\bar{\mu}_{p}$ is shown as obtained from Eq. 5 , see the red line in Figure 11(b). Note that $\bar{\mu}_{p}\left(x_{c}=1\right)<\bar{\mu}_{p}\left(x_{c}=0\right)$ because cosolvent is the better of the two (co)solvents. Furthermore the behavior of $\mathcal{V}$ presented in Figure 11 is consistent with the $\bar{\mu}_{p}$ for $\chi_{s c}<0$ shown by a hump for the intermediate mixing ratios, thus the solvent quality goes from good-to-poor-to-good again. It should also be noted that the trend in Figure 10 is qualitatively different from what is expected from the Flory-Huggins theory. In the mean-field picture, however, when $\chi_{s c}>>0$ similar trend as in Figure 10 be expected, see the black curve in Figure 11(b). Moreover, this will imply that the trend in $\bar{\mu}_{p}$ is obtained at the non-realistic cost of driving the system towards solvent phase separation. In this context, it was already noticed early (18) that for common solvent mixtures where co-non-solvency effects are observed, such as water-alcohol mixtures, $\chi_{s c} \geq 0$.

3.2.2. Co-non-solvency as a result of cooperativity effects. A theoretical approach for describing the LCST behavior of PNIPAm was developed earlier (111), based on two central ideas: i) water molecules bind to the PNIPAm backbone through hydrogen bonds, in a cooperative manner: the formation of bonds between one water molecule and the monomer facilitates the formation of the next bonds, and ii) the sections of the backbone without bound water molecules are naturally in a collapsed state while the sections with bound water molecules are naturally in good solvent conditions. An extension of this model for co-non-solvency was then considered (24) where both water and cosolvent were treated in the same manner, albeit with possible different values for the parameters measuring affinities and cooperativity. Model parameters can be found that fit well experimental results on the variation of PNIPAm radius of gyration in water-methanol mixtures (24). 
3.2.3. Competitive displacement of solvent by cosolvent. The approach in Ref. (24) successfully displays the non-monotonic collapse behavior of a polymer under con-non-solvency, it can lead to the conclusion that co-non-solvency can only be generated by the the competition between two solvents that cooperatively bind to the chain backbone in a poor solvent. More recently it was shown that the co-non-solvency can be a generic phenomena emerging in much less restrictive conditions.

As demonstrated by the simulations (see Figure 9) the polymer has preferential interactions with the cosolvent molecules. Because of this preferential interaction, when a small amount of cosolvents are added into the solution it tries to minimize the polymer-cosolvent binding free energy by attaching to more than one monomer at a time. Furthermore, the molecular flexibility of a polymer can help in this cause by forming segmental loops when cosolvent molecules form contact between two monomers far along the polymer backbone. Note- within the simplified generic model, one cosolvent sphere does not necessarily correspond to one alcohol molecule, rather a collections of several alcohol molecules.

The general picture of cosolvent molecules forming bridging interactions between two monomers topologically far along the polymer backbone has also been proposed for polymer collapse in a broad range of aqueous cosolvent mixtures. For example, the collapse of a PNIPAm in aqueous urea mixtures was shown to be driven by bridging-like hydrogen bonding of urea with two NIPAm monomers $(15,35)$. Furthermore, there are also other works showing that bridging interactions are responsible for a polymer collapse in a mixtures of two cosolvents $(36,37)$, while another work highlighted that the preferential binding may not be prerequisite for co-non-solvency (109).

In view of this, a non Flory-Huggins mean-field description of polymer solution can be formulated that is based on the Langmuir like adsorption isotherm of competitive displacement (112). Within this theory a polymer is considered as an adsorbing substrate, where $N$ sites are exposed to the bulk solution, of which $n^{s}$ sites are occupied by $s$ (solvent) molecules, $n^{c}$ sites by non-bridging $c$ (co-solvent) molecules and $2 n_{B}^{c}$ sites by bridging $c$ (co-solvent) molecules, with $N=n^{s}+n^{c}+2 n_{B}^{c}$. The observed sequence of collapse and re-swelling of the polymer corresponds to a fast growth of $n_{B}^{c}$ as $x_{c}$ increases, followed by a displacement of $n_{B}^{c}$ by $n^{c}$ for larger $x_{c}$ values. Such a sequence is typical for competitive displacement in adsorption phenomena (112).

The results from numerical simulations for $n_{B}^{c}$ and $n^{c}$, or alternatively for the fractions $\phi_{\mathrm{B}}=n_{B}^{c} / N$ and $\phi=n^{c} / N$, are very well described by a competitive adsorption model with the following associated free energy density of adsorption for non-bridges and bridges (3),

$$
\begin{aligned}
\frac{\Psi}{\kappa_{\mathrm{B}} T} & =\phi \ln (\phi)+\zeta \phi_{\mathrm{B}} \ln \left(2 \phi_{\mathrm{B}}\right) \\
& +\left(1-\phi-2 \phi_{\mathrm{B}}\right) \ln \left(1-\phi-2 \phi_{\mathrm{B}}\right) \\
& -\mathcal{E} \phi-\mathcal{E}_{\mathrm{B}} \phi_{\mathrm{B}}-\frac{\mu}{\kappa_{\mathrm{B}} T}\left(\phi+\phi_{\mathrm{B}}\right)
\end{aligned}
$$

with $\mu=\kappa_{\mathrm{B}} T \ln \left(x_{c}\right)$ being the chemical potential of the cosolvent in the bulk solvent mixture and the adsorption energies $\mathcal{E}$ and $\mathcal{E}_{\mathrm{B}}$ measure the excess affinities of individual non-bridging and bridging cosolvent molecules to the chain monomers. The first three terms in Eq. 6 express entropic contributions of the adsorbed bridges and non-bridges to the energy densities, while the two following terms measures contact energies between the cosolvents bridges and non-bridges with the polymer backbone. The unusual pre-factor $\zeta$ is a consequence of assuming a logarithmic form for the dependence of the energy required to make a bridge on the average density of existing bridges. This is the case for instance 
(3), if one assumes that in order to make a new bridge at density $\phi_{\mathrm{B}}$, the chain needs to make a loop of length $\ell=1 / \phi_{\mathrm{B}}$, with associated penalty $\sim \log \ell \sim \log \left(1 / \phi_{\mathrm{B}}\right)$.

Minimization of Eq. 6 with respect to $\phi_{\mathrm{B}}$ and $\phi$ leads to the implicit equation for the bridge density $\phi_{\mathrm{B}}\left(x_{c}\right)$,

$$
\begin{aligned}
16 \phi_{\mathrm{B}}{ }^{\zeta} x_{c} & =x_{c}^{*}\left\{\left(\frac{x_{c}^{*}}{x_{c}^{* *}}\right)^{1 / 2}\left(1-2 \phi_{\mathrm{B}}\right)\right. \\
& \left. \pm \sqrt{\left(\frac{x_{c}^{*}}{x_{c}^{* *}}\right)\left(1-2 \phi_{\mathrm{B}}\right)^{2}-16 \phi_{\mathrm{B}}{ }^{\zeta}}\right\}^{2} .
\end{aligned}
$$

with $x_{c}^{*}=\exp (-\mathcal{E})$ and $x_{c}^{* *}=\exp \left(-\mathcal{E}_{\mathrm{B}}+2 \ln 2 e-\zeta\right)$ are the characteristic concentrations related to the adsorption energies $\mathcal{E}$ and $\mathcal{E}_{\mathrm{B}}$ for non-bridges and bridges. In Fig. 12(a) we show $\phi_{\mathrm{B}}$, the fraction of backbone sites participating in bridge formation, as a function of $x_{c}$. Note that $\phi$ and $\phi_{\mathrm{B}}$ are cosolvent molecules that are directly in contact with the monomers at a distance $2^{1 / 6} \sigma_{p c} \sim 0.84 \sigma$. As the figure shows expression Eq. 7 describes very well our experimental results, with $\zeta=0.05$.

Figure 12

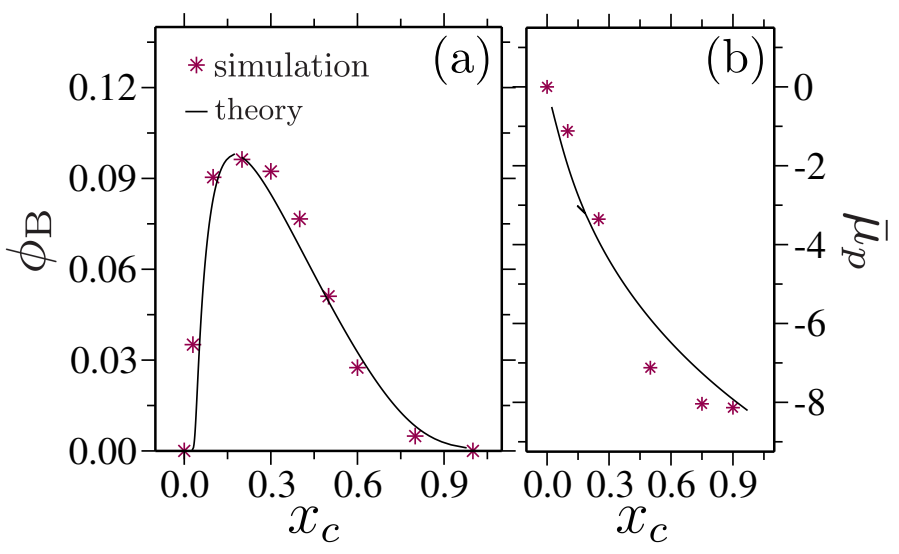

A comparative plot of molecular dynamics simulation (symbols) and theoretical plot (solid line) (3). Part (a) shows bridging fraction of cosolvents $\phi_{\mathrm{B}}$ as a function of cosolvent mole fraction $x_{c}$. Theoretical prediction is represented by Eq. 7. Part (b) shows chemical potential shift $\bar{\mu}_{p}$ with $x_{c}$. Figure is taken from Nature Publishing Group.

Eq. (7) can equivalently be derived by considering the two pseudo chemical reactions

$$
\begin{array}{r}
\text { cosolvent }+ \text { empty site } \rightleftharpoons \text { non }- \text { bridge } \\
\text { cosolvent }+2 \text { empty site } \rightleftharpoons \zeta \text { bridge },
\end{array}
$$

sketched in Figure 13. When the solvent and cosolvent interactions with the polymer backbone empty sites are described as pseudo reactions, a cosolvent molecule reacts with one empty adsorption site to form one adsorbed non-bridge, while it reacts with two empty sites to make $\zeta$ bridges. The associated equilibrium mass-action laws can thus be written as

$$
\begin{aligned}
\frac{x_{c}}{x_{c}^{*}} & =\frac{\phi}{1-\phi-2 \phi_{\mathrm{B}}} \\
\frac{x_{c}}{4 x_{c}^{* *}} & =\frac{\phi_{\mathrm{B}}^{\zeta}}{\left(1-\phi-2 \phi_{\mathrm{B}}\right)^{2}},
\end{aligned}
$$


(a)

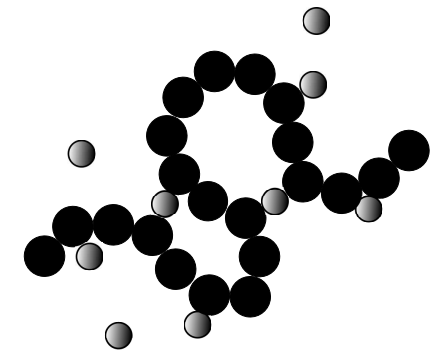

(b)

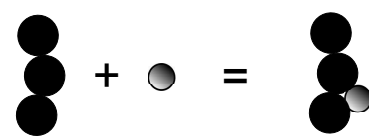

(c)

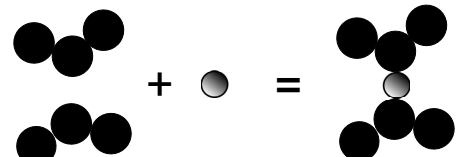

Figure 13

A schematic representation of the chemical reaction described in Eq. 11. Part (a) shows a polymer conformation decorated by non-bridging and bridging cosolvents. Part (b) shows a polymer segment and a cosolvent forming a single non-bridging cosolvent, while part (b) represents two segments making a cosolvent bridge (110). Figure is taken from American Institute of Physics.

with equilibrium reaction constants $1 / x_{c}^{*}$ and $1 / x_{c}^{* *}$, where the reaction equilibrium concentration $x_{c}^{* *}$ has been, for mathematical convenience, defined up to a factor four. Solving the mass-action laws for $\phi_{\mathrm{B}}$ gives Eq. (7). In this pseudo-chemical language, the factor $\zeta$ describing the effective number of bridges formed by the interaction between one cosolvent molecule and the two empty sites of the backbone appears as a consequence of assuming a power-law dependence for the equilibrium constant of the pseudo-chemical reaction. Note that the actual shape of Eq. 7 is quite sensitive to the value of $\zeta$. In particular, the choice $\zeta=1$, corresponding to a standard chemical reaction between free species in solution, leads to a prediction that can not describe our data.

In a previous work (3), it was argued that a value of $\zeta=0.05$ can be understood by considering loop contributions to the cost of making a bridge. When a pure configurational cost for distributing the bridges amongst the possible occupation sites is combined with the entropic cost of loop formation, one can write $\zeta=2-m$. Here the critical exponent $m$ can be estimated within a simple scaling argument. In this context, one can characterize the loop formation by a partition function of vanishing end-to-end distance $R_{e} \rightarrow 0$ (66),

$$
Z_{N_{l}}\left(R_{e} \rightarrow 0\right) \propto q^{N_{l}} N_{l}^{\alpha-2},
$$

and the partition function at finite $R_{e}$ is given by,

$$
Z_{N_{l}}\left(R_{e}\right) \propto q^{N_{l}} N_{l}^{\gamma-1}
$$

Here $1 / q$ is the critical fugacity and the universal exponent $\alpha \cong 0.2$ (66). From these two cases one can estimate the free energy barrier to form a loop of length $\ell$ as $\triangle \mathcal{F}(\ell)=$ $m \kappa_{\mathrm{B}} T \ln (\ell)$, with $m=\gamma-\alpha+1$ being the critical exponent (66). Although this gives 
$m=1.95$ for loop formation in self-avoiding walks, in excellent agreement with our findings, it is worth pointing to the fact that our simple analytical description does not address other possible contributions to bridge formation, such as cooperative or other non-trivial entropic effects that might be determinant in the dense chain globule.

This selective adsorption model provides also for an analytical prediction of the shift in the chemical potential $\mu_{p}$ as a function of $x_{c}$,

$$
\begin{aligned}
\frac{\mu_{p}}{\kappa_{\mathrm{B}} T} & =\text { const }+(2-\zeta) \phi_{\mathrm{B}} \\
& -\ln \left\{1+\phi_{\mathrm{B}}{ }^{1-\zeta / 2}\left(\frac{x_{c}}{x_{c}^{* *}}\right)^{1 / 2}+\left(\frac{x_{c}}{x_{c}^{*}}\right)\right\} .
\end{aligned}
$$

Fig. 12(b) shows a comparison between predictions from Eq. 14 and the values of the chemical potential obtained from Eq. 14. A very good agreement is obtained by simply inserting into Eq. 14 the values for $\zeta$ and concentrations obtained from the fit of the bridging fraction, further confirming the consistency and validity of our approach.

Note that while standard Flory-Huggins type mean field theory does not describe conon-solvency, mean-field descriptions with higher order corrections may be more applicable. In this context, a recent extension of the competitive displacement concept $(3,110)$ led to the introduction of sticky sites within a mean-field picture to describe co-non-solvency of polymer brushes $(113,114)$.

\subsection{UCST-like swelling of LCST polymer}

It is commonly known that a PNIPAm chain collapses in water upon increase of $T$, thus showing a LCST like temperature effect. Moreover, in aqueous alcohol mixtures, especially for larger alcohol (such as ethanol or propanol), PNIPAm also shows UCST-like swelling with increasing $T(69,31,115)$. As shown in Figure 14 both simulation and experiments
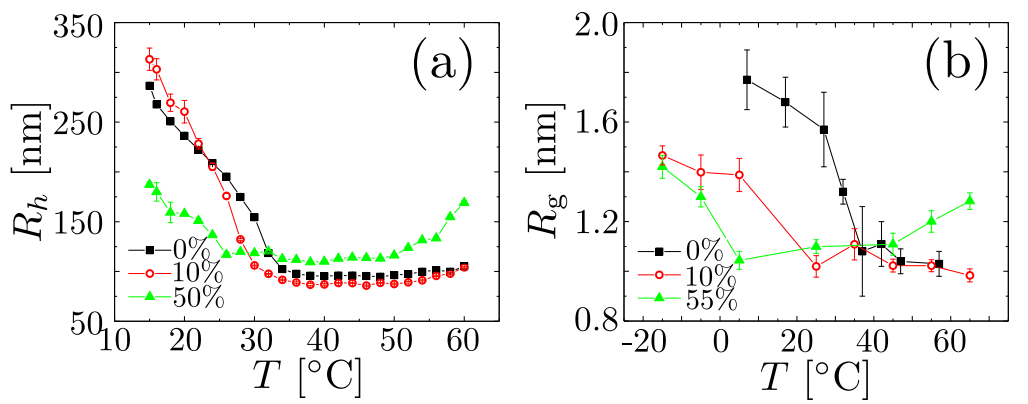

Figure 14

Part (a) shows hydrodynamic radius $R_{h}$ obtained from dynamics light scattering of PNIPAm microgel and part (b) for all-atom molecular dynamics simulations of single PNIPAm chain, respectively. Data is shown fro three different ethanol volume fractions and with varying temperature $T$ (69). Figure is taken from American Chemical Society.

show that the UCST-like re-swelling is more prominent for ethanol volume fraction $\phi_{e}>$ $50 \%$ (69). This can be attributed to the fact that for $T>305 \mathrm{~K}$ pure water is always a poor solvent for PNIPAm, while no such LCST behavior is known for pure alcohol. Therefore, for $\phi_{e}>50 \%$, alcohol acts as addition of good solvent in poor solvent, thus results in re-swelling. There are of-course other mechanism proposed for such UCST-reswelling via kosmotropic effects (31) and cooperative hydrogen bonded effects (115). 


\section{CO-SOLVENCY: POLYMER SWELLING IN MISCIBLE POOR SOLVENTS}

In the previous section we reviewed the phenomenon of co-non-solvency that describes polymer collapse in a mixtures of two competing, (fairly) miscible good solvents, while the same polymer remains expanded in these two solvents individually. An opposite effect is the swelling of a polymer in mixtures of two fairly miscible poor solvents. In this context, it has been commonly observed that a polymer may remain collapsed in two different poor solvents, whereas it is somewhat "better" soluble in their mixtures $(116,117,118$, 119). This phenomenon known as co-solvency will be discussed in this section. Typical systems include- poly(methyl methacrylate) (PMMA) $(116,117,118,119,120)$, poly (N-(6acetamidopyridin-2-yl)acrylamide) (PNAPAAm) (121), and corn starch in solvent mixtures (122). For example, both water and alcohol are poor solvents for PMMA, it swells within the intermediate mixing ratios of water-alcohol mixtures (such as the aqueous methanol, ethanol and propanol, respectively). Attaining a maximum degree of swelling around 60$70 \%$ alcohol mole fraction, see Figure 15.

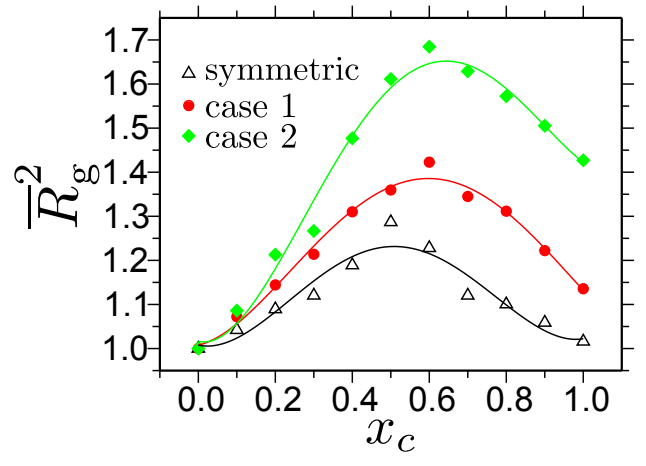

Figure 15

Normalized squared radius of gyration $\bar{R}_{\mathrm{g}}^{2}=\left\langle R_{\mathrm{g}}^{2}\right\rangle /\left\langle R_{\mathrm{g}}\left(x_{c}=0\right)^{2}\right\rangle$ as a function of cosolvent molar concentration $x_{c}$. Results are shown for the generic simulations and for three different cases. Here $\left\langle R_{\mathrm{g}}\left(x_{c}=0\right)^{2}\right\rangle=2.6 \pm 0.4 \sigma^{2}$ and $\bar{R}_{\Theta}^{2}=2.13$ with $\bar{R}_{\Theta}=R_{\Theta} / R_{\mathrm{g}}\left(x_{c}=0\right)$ is the normalized $\Theta$-point gyration radius. Here case 2 closely mimics the conformational behavior of PMMA in aqueous methanol mixture (6). Figure is taken from Nature Publishing Group.

When a polymer collapses in a poor solvent, this collapsed structure is described by balancing negative second virial osmotic contributions $-|\mathcal{V}|$ and three body repulsions. Under such a poor solvent condition, the effective attraction between the monomers of a polymer can be viewed as a depletion induced attraction, a phenomenon well described for colloidal suspensions $(123,124,125,126,127)$ of purely repulsive particles. More specifically, monomer-monomer attraction will occur when monomer-solvent excluded volume interactions become larger than the monomer-monomer excluded volume interactions. The resulting isolated polymer conformation can be well described by the Porod scaling law of the single chain static structure factor $S(q) \propto q^{-4}$ presenting a compact spherical globule, see Figure 16(a). This argument holds in pure water and in pure alcohol for PMMA. Furthermore, the extent of depletion induced attraction between two particles is dictated by the number density $\rho$ of depletants, in this case solvent particles consisting of water and alcohol, within the solvation volume (6). Data that support this view is given by the total number density $\rho_{\text {total }}$ of bulk solution as a function of mixing ratio of two solvents (6). In Figure 17 it is shown that for aqueous alcohol mixtures $\rho_{\text {total }}$ reduces from its mean-field 
Figure 16

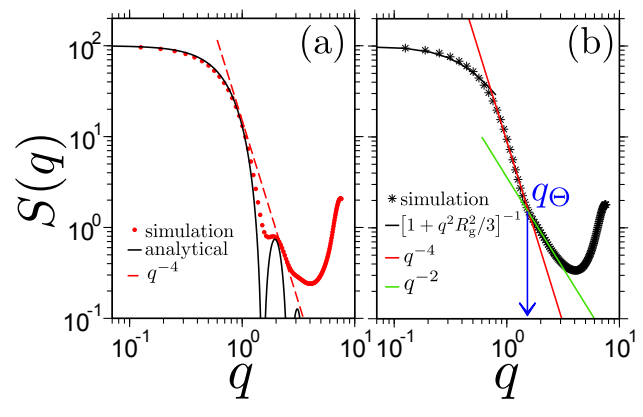

Single chain static structure factor $S(q)$ for: $x_{c}=0.0$ in part (a) and $x_{c}=0.7$ in part (b). In part (a) analytical expression for sphere scattering is included. In part (b) red and green lines are power law fits to the data at different length scales. The black line represents the Guiner region for $q \rightarrow 0$. The vertical arrow indicates the effective $\Theta$-like blob size at $q=q_{\Theta}$ (6). Figure is taken from Nature Publishing Group.

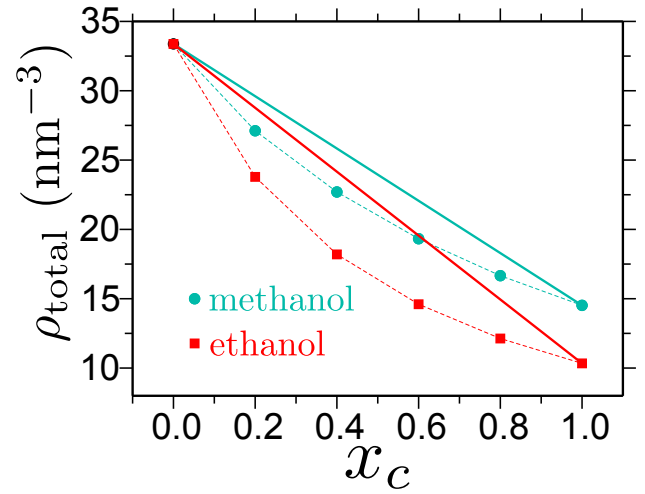

Figure 17

Total number density $\rho_{\text {total }}$ of the bulk solution as a function of mole fraction $x_{c}$ of aqueous methanol and aqueous ethanol solutions, respectively. Solid lines are linear interpolation between the data points of $x_{c}=0.0$ and $x_{c}=1.0$ (128). Figure is taken from Institute of Physics.

value (linear extrapolation between pure solvent $x_{c}=0.0$ and pure cosolvent $x_{c}=1.0$ ) with a maximum deviation observed for the 50-50 mixing ratio $(96,128)$. The larger the alcohol the larger the deviation from the linear density of interpolation. This deviation is a key factor that reduces the number of depletants within the solvation volume and thus reduces the depletion induced attractive forces, reducing the magnitude of the negative excluded volume $\mathcal{V}$. Therefore, the polymer swelling in a mixture of two poor solvents can be viewed as a second order effect. For example, the solvent molecules deplete monomers giving rise to the poor solvent condition for a polymer. However, when cosolvent molecules are added into the system, cosolvents not only deplete monomers but also solvent molecules leading to a second order depletion effect.

As a consequence the mixed solvent remains a poor solvent, while the effective depletion, which drives the polymer collapse is reduced. Thus the observed swelling of about $30-70 \%$ in $R_{\mathrm{g}}^{2}$ (or $10-30 \%$ swelling in $R_{\mathrm{g}}$ ), as observed in Figure 15, does not mean that a polymer is fully swollen into a self avoiding random walk. As seen from Figure 16(b), $S(q)$ shows a 
cross-over scaling: within the range $1.5 \sigma^{-1}<q<3.0 \sigma^{-1}$ an apparent scaling $S(q) \sim q^{-2}$ is observed, which crosses over to $S(q) \sim q^{-4}$ for $0.7 \sigma^{-1}<q<1.5 \sigma^{-1}$, suggesting that the polymer remains globally collapsed consisting of $\Theta$-like blobs. The crossover point $q_{\Theta}$ gives the direct measure of the effective blob size $\ell_{\Theta-\text { blob }}=2 \pi / q_{\Theta} \sim 4.5 \sigma$. The largest blobs are observed when the polymer is maximally swollen.

\subsection{Flory-Huggins mean-field theory}

Unlike the phenomenon of co-non-solvency the phenomenon of co-solvency can be explained within a Flory-Huggins mean-field picture, where the bulk solvent-cosolvent interactions play an important role in dictating solvation properties of polymers. For example, the density dip, as observed in Figure 17, is necessary to observe co-solvency. Here it should also be emphasized that the density dip naturally emerges at constant pressures. Therefore, it is necessary to device a theory that can take the density dip into account. In a recent work (6), it was shown that the co-solvency can be explained within a unified picture combining the knowledge known from polymer physics $(64,65,66)$ and colloid science (123). While a detailed theoretical analysis was presented in (6), here we only sketch a few key ingredients. For simplicity, a single chain at infinite dilution $\phi_{p} \rightarrow 0$ was considered. Up to the single polymer the volume is occupied by solvent-cosolvent mixture. Therefore, the system can be treated within a simplified limit of the binary mixture. In this case, the total free energy can be written as,

$$
\begin{aligned}
\frac{\mathcal{F} v}{\kappa_{\mathrm{B}} T} & =\frac{v \mathcal{F}_{s}(v)}{\kappa_{\mathrm{B}} T}+x_{c} \ln \left(x_{c}\right)+\left(1-x_{c}\right) \ln \left(1-x_{c}\right) \\
& +\chi_{s c}(v) x_{c}\left(1-x_{c}\right)
\end{aligned}
$$

where $\mathcal{F}_{s}(v)$ is the volume-dependent free-energy of the pure solvent of pure cosolvent systems. The Flory-Huggins interaction parameter $\chi_{s c}$ also depends on the system volume or solvent-cosolvent composition. For a given external pressure $P$, the molar volume $v$ is thus controlled by,

$$
P=P_{s}(v)-\kappa_{\mathrm{B}} T x_{c}\left(1-x_{c}\right) \frac{\partial \chi_{s c}(v)}{\partial v}
$$

with $P_{s}(v)=-\partial v \mathcal{F}_{s} / \partial v$ being the pressure of the reference system. For a small variation of the molar volume of the solvent-cosolvent mixture with respect to that of the reference system, one gets $v=v_{\mathrm{o}}\left[1+\zeta x_{c}\left(1-x_{c}\right)\right]$ where

$$
\zeta=\frac{\kappa_{\mathrm{B}} T}{v} \frac{\partial \chi_{s c}(v)}{\partial v}\left[\frac{\partial P_{s}(v)}{\partial v}\right]^{-1}
$$

measures the relative sensitivity of the interaction parameter and reference pressure to $v$. Furthermore, the change in $\chi_{s c}$ between constant density and constant pressure ensembles can be estimated from,

$$
\chi_{s c}(v)=\chi_{s c}\left(v_{\mathrm{o}}\right)+\left.v \frac{\partial \chi_{s c}(v)}{\partial v}\right|_{x_{c} \rightarrow 0} \zeta x_{c}\left(1-x_{c}\right) .
$$

Since $v \partial \chi_{s c}(v) / \partial v \sim \zeta$, this shows that $\chi_{s c}$ obtained between different ensembles is only perturbed to the second order in $\zeta$. For $x_{c}=0.5$, the above equation will lead to a $\sim 11 \%$ 
variation in $\chi_{s c}$ values with respect to the standard values calculated when $\rho_{\text {total }}$ is kept constant (6). Even though $11 \%$ may sound like a small number, this is enough to induce polymer swelling in poor solvent mixtures. Note that in this case swelling does not mean a polymer undergoes a globule-to-coil transition, but experience a rather slight swelling as seen in Figure 16.

\section{SMART POLYMERS FOR MATERIALS DESIGN}

Polymers are everywhere in our every day life, finding uses ranging from physics to materials science and chemistry to biology $(1,4,5)$. In this context, so far we have emphasized the need to develop fundamental understanding of smart polymer properties in single and/or multi-component solvents. Moreover, discussions presented above also aim at the future directions for operational understanding and functional design principles of smart materials. For this purpose, molecular simulations are of particular importance in interpreting and guiding experiments to new directions. Therefore, we aim to finish this review sketching a few examples where the knowledge discussed so far can be used to propose a design principle of polymeric materials.

\subsection{Design of multi-responsive copolymer architectures}

In section 2.1.1 a discussion is presented related to a sequence transferable CG model for polyacetal based copolymer architectures $(13,81)$. While varying parameter space with

Figure 18

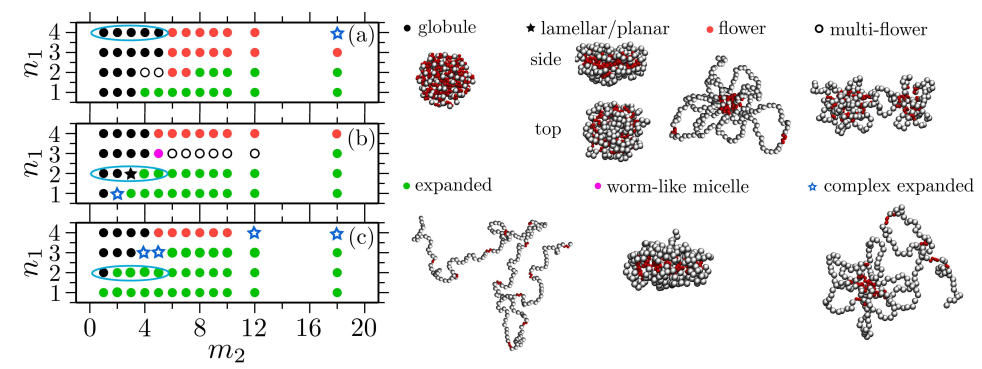

A representative phase diagram of the amphiphilic copolymers with different sequences, see Figure 3. Every symbol in these figures represent one configuration with the color code consistent with the configurations presented in the right column. The data is shown for (a) $m_{1}=0$, (b) $m_{1}$ $=1$, (c) $m_{1}=2$, and (d) $m_{1}=3$ with $n_{2}=2$ and varying $m_{2}$ and $n_{1}$. Ethylene oxide beads are rendered in silver and methylene units are represented by red spheres. Figure is taken from American Institute of Physics.

different sequences is rather non trivial in experiments, the CG model can be used to predict a much broader range of polymer architectures. In Figure 18 a representative phase diagram for 192 polymer sequences are shown. Several interesting structures are observed reminiscent of the poly-soap collapse $(129,130,131)$.

While conformations presented in Figure 18 are shown for statistical copolymers, there are also studies showing nice thermal switching of micellar structures in pure water based on di- (or tri-)block copolymer architectures $(11,132,133)$. Moreover, sometimes thermal switching requires a temperature change much above normal human body temperature. This often restricts broader applications of proposed polymer architectures for biomedi- 
cal encapsulations. An alternative may, therefore, be to use (co-)solvents as a switchable stimulus at a fixed $T$, preferably around ambient temperatures. Here the phenomenon of co-non-solvency (see Section 3) can serve as an ideal platform to tune polymer conformation for desired application. More specifically, if di-block copolymer architectures are designed such that different monomer units of a chain show distinct responsiveness in the same solvent-cosolvent mixtures $(68,134,135)$, one can expect to see interesting structures. One possible example may be a di-block consisting of PNIPAm and PMPC. The specific choice of these monomer structures are because both PNIPAm and PMPC shows co-non-solvency in aqueous alcohol mixtures, while PNIPAm collapses between 10-40\% and PMPC between $50-90 \%$ alcohol mixing ratios, respectively. A comparative figure showing conformation of a $\mathrm{p}$ (NIPAm-co-MPC) in aqueous ethanol mixtures is shown in Figure 19.

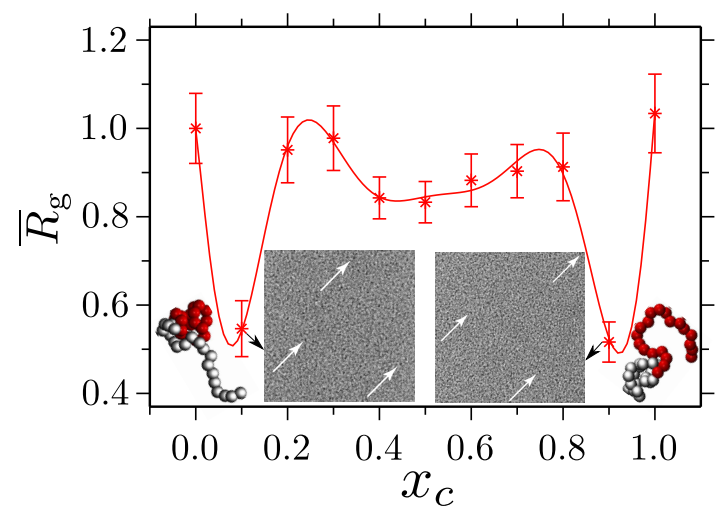

Figure 19

Main panel shows the normalized gyration radius $\bar{R}_{\mathrm{g}}=R_{\mathrm{g}} / R_{\mathrm{g}}^{\circ}$ as a function of cosolvent mole fraction $x_{c}$ obtained from generic molecular simulations cite us. Two minima around $x_{c}=0.1$ and 0.9 are because of the collapse of one block, as shown by the simulation snapshots. Inset also shows cryo-TEM images indicating the presence of micellar objects (135). Arrows in the cryo-TEM images indicate at the region of maximum contrast.

The generic molecular simulations predict a bimodal conformational transition (see the main panel in Figure 19), while cryo-TEM also suggests micellar structures for $x_{c}=0.1$ and 0.9 (see the insets in Figure 19). These interesting conformations show that solventcosolvent composition can induce switchable micellization.

\subsection{Polymers with improved thermal properties}

Another interesting application of hydrogen bonded polymers is their possible use for the improved (tunable) thermal properties of polymeric materials $(55,56,136,137)$. In this context, while polymers are versatile and widely used, one drawback that often limits their usefulness under high temperature conditions is their poor thermal conductivity $\kappa$. Most commonly known polymers, namely polystyrene, polypropylene, poly-carbonate and PMMA to name a few, show $\kappa$ values that are below $0.2 \mathrm{~W} / \mathrm{K}-\mathrm{m}(136)$. This is because the dominant interactions in these systems is of the order of $k_{\mathrm{B}} T$. One standard protocol to improve $\kappa$ of polymers is to blend them with high $\kappa$ materials, such as the carbon based materials that show $\kappa$ exceeding above normal metals. Moreover, significant improvement 
in $\kappa$ often requires blending concentrations exceeding their percolation threshold. A more attractive protocol, therefore, is to use monomer chemistry that show stronger interaction between monomer segments. Here water soluble polymers are of particular important, where dominant interaction is hydrogen bonding and the strength of whose ranges from 4-8 $k_{\mathrm{B}} T$ depending on temperatures and dielectric constants of the medium.

Figure 20

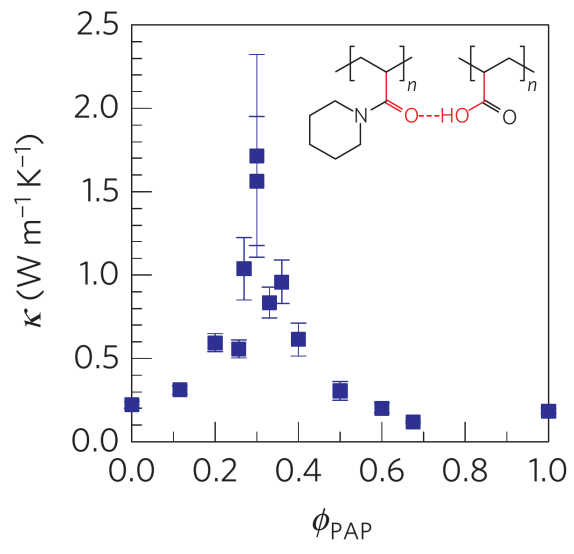

Thermal transport coefficient $\kappa$ of poly(acrylic acid) (PAA) and poly ( $N$-acryloyl piperidine) (PAP) blend as a function of PAP monomer mole fractions $\phi_{\text {PAP }}(55)$. Figure is taken from Nature Publishing Groups.

There are recent interests to study thermal properties of water soluble polymers in their dry states $(55,56)$. This reaches from the homopolymer to copolymers and to polymer blends. For example, hydrogen bonded homopolymer systems shows $\kappa \sim 0.4 \mathrm{~W} / \mathrm{K}-\mathrm{m}$ (56). In particular, when two hydrogen bonded polymers are blended in, $\kappa$ could be tuned with their typical values exceeding $1.5 \mathrm{~W} / \mathrm{K}-\mathrm{m}(55)$. One of the experimentally relevant systems is asymmetric blend of a long poly(acrylic acid) (PAA) and short poly $(N$-acryloyl piperidine) (PAP), see Figure 20. In a separate study, however, no such enhancement was observed for the PAA-PAP blend, while the the system phase separate over almost full concentration range of $\phi \mathrm{PAP}(56,138)$

\section{SUMMARY}

Over the last three decades the field of smart polymer research has grown enormously, and there is now an exciting body of literature about this class of polymers. In this short overview we have reviewed a facet of smart polymer research related to the solvation thermodynamics of hydrogen bonded polymers in mostly aqueous mixtures. In particular, we have discussed two symmetric, yet distinct, phenomena of polymers in miscible solvent mixtures: co-non-solvency and co-solvency. We have put these phenomena into perspective by a combination of multi-scale modeling with complimentary experiments and analytical theories. It is discussed how the changes in solution behavior or morphology of polymer systems emerge as a function of external driving forces, such as the changes in temperature, concentration or additives and subject to (small) chemical variations. We highlighted here 
the more recent unified frameworks that allow researchers to combine different concepts in order to arrive at tailor made properties.

\section{DISCLOSURE STATEMENT}

The authors are not aware of any affiliations, memberships, funding, or financial holdings that might be perceived as affecting the objectivity of this review.

\section{ACKNOWLEDGMENTS}

We have reviewed several results that were obtained within fruitful collaborations with many colleagues, especially - Torsten Stühn, Paulo Netz, Tiago Oliveira, Manfred Wagner, Mark Watson, Marc Schmutz, Svenja Morsbach, Takahiro Ohkuma, Chathuranga De Silva, Porakrit Leophairatana, Jeffrey Koberstein, Sebastian Backes, Regine von Klitzing, David Ng, Tanja Weil, Daniel Bruns and Jörg Rottler- which we take this opportunity to gratefully acknowledge. Furthermore, this work has greatly benefited from stimulating discussions with Burkhard Dünweg, Martin Müser, Kostas Ch. Daoulas, Robinson Cortes-Huerto, Christine Rosenauer, and Robert Graf. K.K. acknowledges the support by the European Research Council under the European Union's Seventh Framework Programme (FP7/20072013)/ERC Grant Agreement No. 340906-MOLPROCOMP. We further thank Robinson Cortes-Huerto and Tapan Chandra Adhyapak for the critical reading of this manuscript.

\section{LITERATURE CITED}

1. Cohen-Stuart MA, Huck WTS, Genzer J, Müller M, Ober C, Stamm M, Sukhorukov GB, Szleiferi I, Tsukruk VV, Urban M, Winnik F, Zauscher S, Luzinov I, Minko S. 2010. Nature Materials 9:101.

2. de Beer S, Kutnyanszky E, Schön PM, Vancso GJ, Müser MH. 2014. Nat. Commun. 5:3781.

3. Mukherji D, Marques CM, Kremer K. 2014. Nat. Commun. 5:4882.

4. Halperin A, Kröger M, Winnik FM. 2015 Angew. Chem. Int. Ed. 54:15342.

5. Zhang Q, Hoogenboom R. 2015. Prog. Polym. Science 48:122.

6. Mukherji D, Marques CM, Stühn, Kremer K. 2017. Nat. Commun. 8:1374.

7. Wu C, Wang X. 1998. Phys. Rev. Lett. 80:4092.

8. Wang X, Qui X, Wu C. 1998. Macromolecules 31:2972.

9. Meyer DE, Chilkoti A. 1999. Nat. Biotechnol. 17:1112.

10. Li C, Buurma NJ, Haq I, Turner C, Armes SP, Castelletto V, Hamley IW, Lewis AL. 2005. Langmuir 21:11026.

11. Lutz JF, Akbemir Ö, Hoth A. 2006. J. Am. Chem. Soc. 128:13046.

12. Cui S, Pang X, Zhang S, Yu Y, Ma H, Zhang X. 2012. Langmuir 28:5151.

13. Samanta S, Bogdanowicz DR, Lu HH, Koberstein JT. 2016. Macromolecules 49:1858.

14. Zhang M, Jia Y-G, Liu L, Li J, Zhu XX. 2018. ACS Omega 3:10172.

15. Zhang Y, Furyk S, Bergbreiter DE, Cremer PS. 2005. J. Amer. Chem. Soc. 127:14505.

16. Sakota K, Tabata D, Sekiya H. 2015. J. Phys. Chem. B 119:10334.

17. Okur HI, Hladilkova J, Rembert KB, Cho Y, Heyda J, Dzubiella J, Cremer PS, Jungwirth P. 2017. J. Phys. Chem. B 121:1997.

18. Schild HG, Muthukumar M, Tirrell DA. 1991. Macromolecules 24:948.

19. Winnik FM, Ringsdorf H, Venzmer J. 1990. Macromolecules 23:2415.

20. Zhang G, Wu C. 2001. Phys. Rev. Lett. 86:822.

21. Hiroki, A, Maekawa Y, Yoshida M, Kubota K, Katakai R. 2001. Polymer 42:1863. 
22. Kiritoshi Y, Ishihara K. 2002. J. Biomater. Sci., Polym. Ed. 13:213.

23. Kiritoshi Y, Ishihara K. 2003. Sci. Technol. Adv. Mater. 4:93.

24. Tanaka F, Koga T, Winnik FM. 2008. Phys. Rev. Lett. 101:028302.

25. Sagle LB, Zhang Y, Litosh VA, Chen X, Cho Y, Cremer PS. 2010. J. Amer. Chem. Soc. 131:9304.

26. Tanaka F, Koga T, Kojima H, Xue N, Winnik FM. 2011. Macromolecules 44:2978.

27. Kojima H, Tanaka F, Scherzinger C, Richtering W. 2012. J. Polym. Sci. B 51:1100.

28. Walter J, Sehrt J, Vrabec J, Hasse H. 2012. J. Phys. Chem. B 116:5251.

29. Heyda J, Muzdalo A, Dzubiella J. 2013. Macromolecules 46:1231.

30. Mukherji D, Kremer K. 2013. Macromolecules 46:9158.

31. Bischofberger I, Calzolari DCE, Trappe V. 2014. Soft Matter 10:8288.

32. Dudowicz J, Freed KF, Douglas JF. 2015. J. Chem. Phys. 143:131101.

33. Rodriguez-Ropero F, Hajari T, van der Vegt NFA. 2015. J. Phys. Chem. B 119:15780.

34. Kyriakos K, Philipp M, Lin C-H, Dyakonova M, Vishnevetskaya N, Grillo I, Zaccone A, Miasnikova A, Laschewsky A, Müller-Buschbaum P, Papadakis CM. 2016. Macromol. Rapid Commun. 37:420.

35. Micciulla S, Michalowsky J, Schroer MA, Holm C, von Klitzinga R, Smiatek J. 2016. Phys. Chem. Chem. Phys. 18:5324.

36. Zhu P-W, Chen L. 2019. Phys. Rev. E 99:022501.

37. Perez-Ramirez HA, Haro-Prez C, Vzquez-Contreras E, Klapp J, Bautista-Carbajald G, Odriozola G. 2019. Phys. Chem. Chem. Phys. 21:5106.

38. Bedard MF, De Geest BG, Skirtach AG, Möhwald H, Sukhorukov GB. 2010. Adv. Coll. Int. Sci. 158:2

39. Ishii N, Obeid R, Qiu XP, Mamiya J, Ikeda T, Winnik FM. 2010. Mol. Cryst. Liq. Cryst. 527:60.

40. Ishii N, Mamiya J, Ikedaa T, Winnik FM. 2011. Chem. Comm. 47:1267.

41. Esser-Kahn AP, Odom SA, Sottos NR, White SR, Moore JS. 2011. Macromolecules 44:5539.

42. 2012. Macromolecules 45:3647.

43. Kitayama Y, Yoshikawa K, Takeuchi T. 2016. Langmuir 32:9245.

44. Löwe C, Weber C. 2002. Adv. Mat. 14:1625.

45. Caruso MM, Davis DA, Shen Q, Odom SA, Sottos NR, White SR, Moore JS. 2009. Chem. Rev. 109:5755.

46. Bruns N, Pustelny K, Bergeron LM, Whitehead TA, Clark DS. 2009. Angewandte Chem. 48:5666.

47. HernandezSosa G, Bornemann N, Ringle I, Agari M, Dörsam E, Mechau N, Lemmer U. 2013. Adv. Func. Mat. 23:3164.

48. Yablonovitch E. 1987. Phys. Rev. Lett. 58:2059

49. John S. 1987. Phys. Rev. Lett. 58:2486.

50. Fudouzi H, Xia Y. 2003. Langmuir 19:9653.

51. Yin SN, W CF, Liu SS, Chen S. 2013. J. Mat. Chem. C 1:4685.

52. Montarnal D, Capelot M, Tournilhac F, Leibler L. 2011. Science 334:965.

53. Brutman JP, Delgado PA, Hillmyer MA. 2014. ACS Macro Letters 3:607.

54. Röttger M, Domenech T, van der Weegen R, Breuillac A, Nicolaÿ R, Leibler L. 2017. Science 356:62.

55. Kim G, Lee D, Shanker A, Shao L, Kwon MS, Gidley D, Kim J, Pipe KP. 2015. Nat. Mat. $14: 295$

56. Xie X, Li D, Tsai T, Liu J, Braun PV, Cahill DG. 2016. Macromolecules 49:972.

57. Adams ML, Lavasanifar A, Kwon GS. 2003. J. Pharma. Sci. 92:1343.

58. Chang DP, Dolbow JE, Zauscher S. 2007. Langmuir 23:250.

59. Lee H, Lee BP, Messersmith PB. 2007. Nature 448:338.

60. Batrakova EV, Kabanov AV. 2008. J. Cont. Rel. 130:98. 
61. Schmidt S, Zeiser M, Hellweg T, Duschl C, Fery A, Möhwald, H. 2010. Adv. Funct. Mater. 20:3235.

62. Vogel MJ, Steen PH. 2010. Proc. Natl. Acad. Sci. U.S.A. 107:3377.

63. Meddahi-Pelle A, Legrand A, Marcellan A, Louedec L, Letourneur D, Leibler L. 2014. Angew. Chem., Int. Ed. 53:6369.

64. de Gennes PG. 1979. Scaling Concepts in Polymer Physics Cornell University Press.

65. Doi M, Edwards SF. 1986. The Theory of Polymer Dynamics Oxford University Press.

66. Des Cloizeaux J, Jannink G. 1990. Polymers in Solution: Their Modelling and Structure Clarendon Press.

67. Kratz K, Hellweg T, Eimer W. 2000. Coll. Surf. A: Phys. Eng. Aspects 170:137.

68. Scherzinger C, Lindner P, Keerl M, Richtering W, 2010. Macromolecules 43:6829.

69. Backes S, Krause P, Tabaka W, Witt MU, Mukherji D, Kremer K, von Klitzing R. 2017. ACS Macro Lett. 6:1042.

70. Landau LD, Lifshitz EM. 2003. Statistical Physics 3rd Edition Elsevier ButterworthHeinemann.

71. Jeppesen C, Kremer K. 1996. Europhys. Lett. 34:563.

72. Kawasaki H, Nakamura T, Miyamoto K, Tokita M, Komai T. 1995. J. Chem. Phys 103:6241.

73. de Oliveira TE, Marques CM, Netz PA. 2018. Phys. Chem. Chem. Phys. 20:10100.

74. Gernandt J, Frenning G, Richtering W, Hansson P. 2011. Soft Matter 7:10327.

75. Ray B, Okamoto Y, Kamigaito M, Sawamoto M, Seno K, Kanaoka S, Aoshima S. 2005. Polym. $J 37: 234$.

76. Hirano T, Okumura Y, Kitajima H, Seno M, Sato T. 2006. J. Polym. Sc. A: Polym. Chem. 44:4450.

77. de Oliveira TE, Mukherji D, Kremer K, Netz PA. 2017. J. Chem. Phys. 146:034904.

78. Hoffman AS et al. 2000. J. Biomed. Mater. Res. 52:577.

79. Shen Z, Terao K, Maki Y, Dobashi T, Ma G, Yamamoto T. 2006. Colloid Polym. Sci. 284:1001.

80. Schulz B, Chudoba R, Heyda J, Dzubiella J. 2015. J. Chem. Phys. 143:243119.

81. De Silva CC, Leophairatana P, Ohkuma T, Koberstein JT, Kremer K, Mukherji D. 2017. J. Chem. Phys. 147:064904.

82. Tschöp W, Kremer K, Batoulis J, Bürger T, Hahn O. 1998. Acta. Polym. 49:61.

83. Tschöp W, Kremer K, Batoulis J, Bürger T, Hahn O. 1998. Acta. Polym. 49:75.

84. Reith D, Pütz M, Müller-Plathe F. 2003. J. Comput. Chem 24:1624.

85. de Oliveira TE, Netz PA, Kremer K, Junghans C, Mukherji D. 2016. J. Chem. Phys. 144:174106.

86. Wu Y, Ng DYW, Kuan SL, Weil T. 2014. Biomater. Sci. 3:214.

87. Weinberger A, Walter V, MacEwan, SR, Schmatko T, Muller P, Schroder AP, Chilkoti A, Marques CM. 2017. Sci. Rep. 7:43963.

88. Riegger A, Chen C, Zirafi O, Daiss N, Mukherji D, Walter K, Tokura Y, Stoeckle B, Kremer K, Kirchhoff F, Ng DYW, Hermann PC, Münch J, Weil T. 2017. ACS Macro Lett. 6:241.

89. Abbott LJ, Stevens MJ. 2015. J. Chem. Phys. 143:244901.

90. Shinoda W, DeVane R, Klein ML. 2007. Mol. Simul. 33:27.

91. Marrink SJ, Tieleman DP. 2013. Chem. Soc. Rev. 42:6801.

92. Mukherjee B, Delle Site L, Kremer K, Peter C. 2012. J. Phys. Chem. B 116:8474.

93. Wolf BA, Willms MM. 1978. Makromol. Chem. 179:2265.

94. Di J, Zuo T, Sarah R, Cheng H, Hammouda B, Han CC. 2016. Macromolecules 49:5152.

95. Di J, Murugappan M, Cheng H, Han CC, Hammouda B. 2017. Macromolecules 50:7291.

96. Perera A, Sokolic F, Almasy L, Koga Y. 2006. J. Chem. Phys. 124:124515.

97. Mukherji D, van der Vegt NFA, Kremer K, Delle Site L. 2012. J. Chem. Theor. Comp. 8:375.

98. Krüger P, Schnell SK, Bedeaux D, Kjelstrup S, Vlugt TJH, Simon JM. 2012. J. Phys. Chem. Lett. 4:235.

99. Ben-Naim A. 2006. Molecular Theory of Solutions Oxford University Press.

100. Cortes-Huerto R, Kremer K, Potestio R. 2016. J. Chem. Phys. 145:141103. 
101. Heidari M, Kremer K, Cortes-Huerto R, Potestio R. 2018. J. Chem. Theor. Comp. 14:3409.

102. Panagiotis PC, Stefanos AD, Panagiotis-Nikolaos T, Theodorou DN. 2019. J. Phys. Chem. B 123:247.

103. Frenkel D, Smit B. 2002. Understanding Molecular Simulations 2nd Edition Academic Press: New York.

104. Praprotnik M, Delle Site L, Kremer K. 2005. J. Chem. Phys. 123:224106.

105. Mukherji D, Wagner M, Watson MD, Winzen S, de Oliveir TE, Marques CM, Kremer K. 2016. Soft Matter 12:7995.

106. Mukherji D, Kremer K. 2017. Polym. Sc. Ser. C 59:119.

107. Magda JJ, Fredrickson GH, Larson RG, Helfand E. 1988. Macromolecules 21:726.

108. Winnik FM, Ottaviani MF, Bossmann SH, Garcia-Garibay M, Turro NJ. 1992. Macromolecules 25:6007.

109. Wang J, Wang N, Liu B, Bai J, Gong P, Rua G, Feng J. 2017. Phys. Chem. Chem. Phys. 19:30097.

110. Mukherji D, Marques CM, Stuehn T, Kremer K. 2015. J. Chem. Phys. 142:114903.

111. Okada Y, Tanaka F. 2005. Macromolecules 38:4465.

112. Hill TL. 1986. An introduction to statistical thermodynamics Courier Corporation.

113. Sommer JU. 2017. Macromolecules 50:2219.

114. Sommer JU. 2018. Macromolecules 51:3066.

115. Toshiki F, Shinyashiki N, Yagihara S, Kita R, Tanaka F. 2018. Langmuir 34:3003.

116. Masegosa RM, Prolongo MG, Hernandez-Feures I, Horta A. 1984. Macromolecules 17:1181.

117. Hoogenboom R, Remzi BC, Guerrero-Sanchez C, Hoeppener S, Schubert US. 2010. Aust. J. Chem. 63:1173.

118. Lee SM, Bae YC. 2014. Polymer 55:4684.

119. Yu Y, Kieviet BD, Kutnyanszky E, Vancso GJ, de Beer S. 2015. ACS Macro Lett. 4:75.

120. Wolf BA, Blaum GJ. 1975. J. Polym. Sci. Polym. Phys. Ed. 13:1115.

121. Asadujjaman A, Ahmadi V, Yalcin M, ten Brummelhuis N, Bertin A. 2017. Polym. Chem. $8: 3140$.

122. Galvez LO, de Beer S, van der Meer D, Pons A. 2017. Phys. Rev. E 95:030602.

123. Lekkerkerker HNW, Tuinier R. 1990. Colloids and the Depletion Interaction Clarendon Press, Oxford.

124. Mao Y, Cates ME, Lekkerkerker HNW. 1995. Phys. Rev. Lett. 75:4548.

125. Mao Y, Cates ME, Lekkerkerker HNW. 1995. Physica A 222:10.

126. Crocker JC, Matteo JA, Dinsmore AD, Yodh AG. 1999. Phys. Rev. Lett. 82:4352.

127. Phillips R. et al. 2012. Physical Biology of the Cell 2nd Edition Garland Science.

128. Mukherji D, Marques CM, Kremer K. 2018. J. Phys. Cond. Mat. 30:024002.

129. Borisov OV, Halperin A. 1995. Langmuir 11:2911.

130. Lashewki A. 1995. Adv. Polym. Sci. 124:1.

131. Lee NK, Abrams CF. 2004. J. Chem. Phys. 121:7484.

132. Chengming L, Buurma NJ, Haq I, Turner C, Armes SP, Castelletto V, Hamley IW, Lewis AL. 2005. Langmuir 21:11026.

133. Sezonenko T, Qiu XP, Winnik FM, Sato T. 2019. Macromolecules 52:935.

134. Kelley EG, Smart TP, Jackson AJ, Sullivana MO, Epps III TH. 2011. Soft Matter 7:7094.

135. Mukherji D, Watson MD, Morsbach S, Schmutz M, Wagner M, Marques CM, Kremer K. 2019. submitted.

136. Choy CL. 1977. Polymer 18:984.

137. Shen S, Henry A, Tong J, Zheng R, Chen G. 2010. Nat. Nanotech. 5:251.

138. Bruns D, de Oliveira TE, Rottler J, Mukherji D. 2019. submitted. 